\title{
Toward a unifying constitutive relation for sediment transport across environments
}

\author{
Morgane Houssais ${ }^{1,2}$ and Douglas J. Jerolmack ${ }^{* 1}$ \\ ${ }^{1}$ Department of Earth and Environmental Science, University of Pennsylvania, 251 Hayden Hall, 240 \\ South 33rd Street, Philadelphia, Pennsylvania, 19104, USA \\ ${ }^{2}$ Levich Institute, City College of CUNY, 140th Street and Convent Avenue, New York, NY 10031
}

\begin{abstract}
Landscape evolution models typically parse the environment into different process domains, each with its own sediment transport law: e.g., soil creep, landslides and debris flows, and river bed-load and suspended-sediment transport. Sediment transport in all environments, however, contains many of the same physical ingredients, albeit in varying proportions: grain entrainment due to a shear force, that is a combination of fluid flow, particle-particle friction and gravity. We present a new take on the perspective originally advanced by Bagnold, that views the long profile of a hillsope-river-shelf system as a continuous gradient of decreasing granular friction dominance and increasing fluid drag dominance on transport capacity. Recent advances in understanding the behavior and regime transitions of dense granular systems suggest that the entire span of granular-to-fluid regimes may be accommodated by a single-phase rheology. This model predicts a material-flow effective friction (or viscosity) that changes with the degree of shear rate and confining pressure. We present experimental results confirming that fluid-driven sediment transport follows this same rheology, for bed and suspended load. Surprisingly, below the apparent threshold of motion we observe that sediment particles creep, in a manner characteristic of glassy systems. We argue that this mechanism is relevant for both hillslopes and rivers. We discuss the possibilities of unifying sediment transport across environments and disciplines, and the potential consequences for modeling landscape evolution.
\end{abstract}

\section{Introduction}

Much of the Earth's surface is composed of granular material: soil, mud, sand, pebbles and boulders. Under various combinations of water and gravity, these materials give and flow to evolve landscapes. Decades ago, Bagnold proposed that dry granular flows and fluid suspensions are two limits of a continuum of Earth-material flows, that represent a gradient in sediment:fluid concentration (Bagnold, 1954, 1956). In practice, however, predicting sediment transport rate in even the simplest of circumstances is a daunting task. Consider that dry sand alone may behave as a solid, liquid or gas (Jaeger et al., 1996, MiDi, 2004) — and that the transitions among these regimes are still not well understood - and we begin to appreciate the challenges of incorporating effects such as fluid turbulence, grain stye and shape heterogeneity, pore pressure, and cohesion. Moreover, velocity scales of interest for sediment movement in natural landscapes range from $10^{-10} \mathrm{~m} / \mathrm{s}$ for slow-moving landslides (Di Maio et al., 2013) to $10^{0} \mathrm{~m} / \mathrm{s}$ for grains suspended in rivers (Bouchez et al., 2011) and turbidity currents (Xu, 2010).

${ }^{*}$ Corresponding authm. Email. secilmentissas.upenn.edu

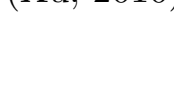


Accordingly, Bagnold's continuum has since splintered into a zoo of transport laws that correspond to different regimes of process dominance (Dietrich et al., 2003) (Fig. 2). To model landscape evolution, the environment is divided up into spatial domains associated with each. In the absence of significant fluid forces, soil "creeps" downhill in a manner akin to a highly viscous fluid and that is modeled using a diffusion equation in which flux is proportional to slope (Culling, 1960, Roering et al., 1999, Smith and Bretherton, 1972). Under sufficiently large pore pressure and/or gravity force, landslides transition to fluidized debris flows that are governed by a shear-rate dependent, viscoplastic rheology (Iverson et al., 2000, Iverson, 1997, Iverson and George, 2014). Farther down the sediment:fluid gradient, river bed-load transport is envisioned as a thin and dense layer of moving grains over the quasi-static bed, where the flux is a power-law function of fluid stress in excess of a critical entrainment stress (Bagnold, 1966 , Barry et al., 2004, Lajeunesse et al., 2010, Meyer-Peter and Müller, 1948, Wong and Parker, 2006). Continuing downstream in the river, sediment moves more and more as a suspension whose concentration results from a balance between upward-directed turbulent diffusion and downward advective settling due to gravity (Rijn, 1984). This type of model has been extended to describe turbidity currents off the continental shelf (Parker et al., 1986).

While much progress has been made, Earth-material flows are often studied in isolation from each other by specialized communities with specific models. More critically, models and experiments focus on one state of flow, rather than transitions between states. For example, there is a mature literature examining the controls on the velocity and concentration profiles of steady-state turbidity currents (Felix and Peakall, 2006, Sequeiros et al., 2009, 2010, Talling et al. 2012) - submarine density currents made of suspended sediment - however there are remarkably few studies documenting how these currents are formed from a collapsing granular pile (You et al., 2012). A similar statement can be made regarding debris flows. The intermittent driving of the landscape by floods, storms and earthquakes, however, indicates that transitions between transport states are the rule. The triggering of failure or flow of sediment presents a particularly delicate state transition. For debris flows, failure is typically treated using a simple yield stress criterion, below which the bed is immobile. This is, however, incompatible with experimental observations of material creep over long times (Barnes, 1999, Mitchell, 1993 , Nguyen et al., 2011). While there may often be an important separation of time scales between creep and debris flows such that a threshold approximation holds, this need not be the case; landslides may transit across these regimes continuously, making the notion of a yield stress a fuzzy concept (Mackey and Roering, 2011). In parallel, defining and assessing the critical stress for particle entrainment is a notorious problem for bed-load transport (Buffington and Montgomery, 1997, Shields, 1936). In principle, one would need to wait an infinitely long time in order to demonstrate a state of no motion. Practically, this means that determinations of threshold are strongly dependent on the method used. Our recent experiments show that fluid-driven sediment transport undergoes a continuous state transition from creep to bed load that is not simply related to a critical fluid shear-stress (Houssais et al., 2015, 2016). Creeping granular motion had only previously been associated with (dry) hillslope transport. Similar to hillslopes, our experiments showed that creep can occur even for stresses below the apparent critical stress. Therefore, on one hand, landslides, undersea avalanches, and the collapse of lava domes all create catastrophic sediment-fluid flows that result from the sudden loss of rigidity of a quasi-static granular system (Fig. 1). On the other hand, state transitions may also be gradual and multiple flow regimes may coexist, as is often the case for bed-and suspended-load transport.

One thing shared by all of the sediment-transport systems described above is that they exhibit an effective viscosity that depends on inter-particle friction and the degree of mixing with the fluid medium. Parallel research in the field of granular and soft-matter physics has 

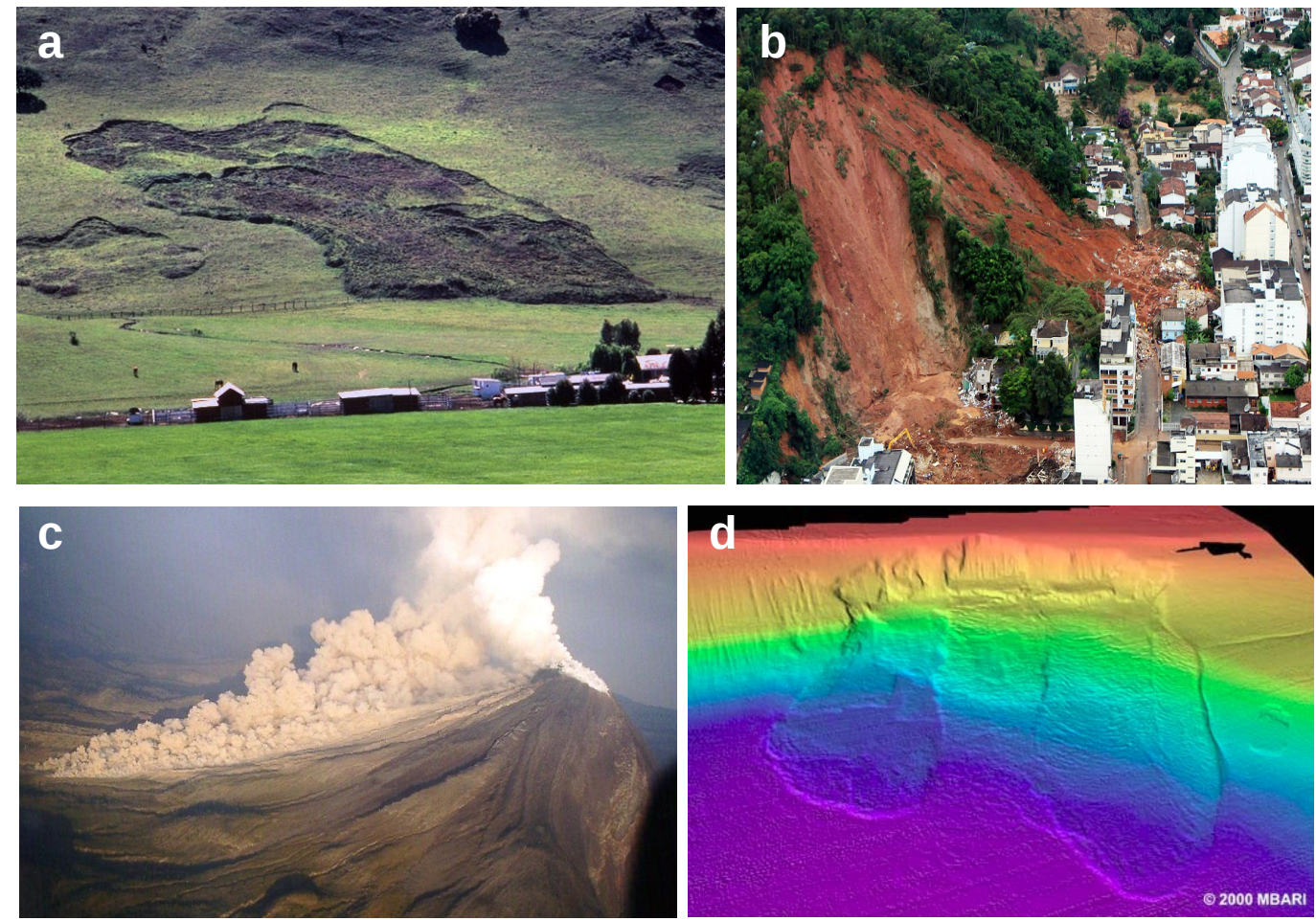

Figure 1: Examples of Earth-material failures and flows involving granular material. a) A slumping, slow-moving landslide that is below the threshold for fluidization (Credit: wallpaper222.com/explore/earthflow). b) A fast-moving fluidized landslide, showing long runout with catastrophic consequences (Credit: www.weatherwizkids.com). c) A pyroclastic flow, in which particles ranging from ash to boulder size are ejected from a volcano and mixed with hot gas, causing a fluidized granular flow to move downslope (Credit: Abel Cortes of the Colima Volcano Observatory). d) A submarine landslide, in which sediment on the continental slope was fluidized to create a debris flow (Credit: Monterey Bay Aquarium Research Institute). 
begun to unify disparate types of particulate flows under a common rheological framework: there is (1) a jamming point, above which the system exhibits a continous transition from a no-flow (infinite viscosity) to flow (finite viscosity) state, where (2) a non-linear and local rheological law describes the flow.

Based on our recent experimental results (Houssais et al., 2016), we propose that Earthmaterial flows rarely (if ever) reach a fully jammed state of no-flow; rather, creep occurs under even very small stresses. As a consequence, we posit that the failure of Earth materials may be mapped to a creep-to-flow transition, and that sediment transport across environments may be unified using a modified granular local rheology.

To pursue this idea and its implications, we first present in the next section a brief overview of recent developments in rheology and the jamming transition of disordered granular systems. We then review some of our own recent experimental work to examine the potential of this approach for sediment transport. This comparison leads to a broader synthesis of granular physics and landscape dynamics across environments, but also presents a host of new challenges that must be addressed to advance landscape modeling further. We close with our view on the most exciting opportunities for future progress.

\section{Granular rheology}

In this paper we will consider granular materials that are composed of hard (inelastic), athermal (larger than colloids) particles and fluid - unless stated otherwise. The system is considered dry if the fluid is a gas, and submerged if it is a liquid.

We present here a brief summary of the generalized local rheology of granular flows, considering only the simplest cases of unimodal and cohesionless sediment in either dry or fully submerged conditions. Although idealized, these conditions may be considered as a starting point for modeling sediment transport in hillslope creep, debris flows, and rivers. In a infinitely wide and steady-state system tilted at a slope $\theta$, we consider a small parcel of material of size $d x \cdot d z$ (see axis orientation on sketches fig. 2), made of fluid of density $\rho$ and particles of density $\rho_{p}$. The force balance on this parcel involves a total tangential force $F_{/ /}$and a total normal force $F_{\perp}$, expressed per unit width (all variables, symbols and units appear in table 1). In nature the normal force is generally the result of hydrostatic pressure: $F_{\perp}(z) \propto \int_{z}^{+\infty} d x \Phi\left(\rho_{p}-\rho\right) g \cos \theta d z$, with $\Phi$ the packing fraction - the volume fraction occupied by particles - and $g$ is gravity. In general, the forces contributing to $F_{/ /}$are the gravity force, $F_{g}(z) \propto d x d z\left(\rho_{p}-\rho\right) g \sin \theta$, and the shear force, $F_{s}=\tau_{x z} d x$, with $\tau_{x z}$ the shear stress associated with the flow of surrounding material (particles and fluid). The importance of gravity varies with the slope angle $\theta$. In the limiting case of an exposed sediment grain resting on a bed or suspended in a liquid flow, the shear force may be entirely hydrodynamic - as is usually assumed to be the case for sediment transport studies. On the other hand, in dry grain flows the shear force results entirely from granular motion.

Before presenting the granular flow rheology for the dry and submerged cases, we introduce the concept of jamming and its connection to the yield criterion.

\subsection{Yielding, Jamming and Metastable States}

It is now recognized that disordered granular systems, like a pile of sand or a suspension, do not undergo an abrupt transition from flowing to rigid, but rather exhibit a continuous transition in which the effective viscosity diverges on approach to the "freezing" point (Stickel and Powell, 2005). In mechanics, this sort of system has been known as non-linear rheology fluids, as they start to exhibit significant plastic deformations for stresses above their yield stress $\tau_{c}$. In the 

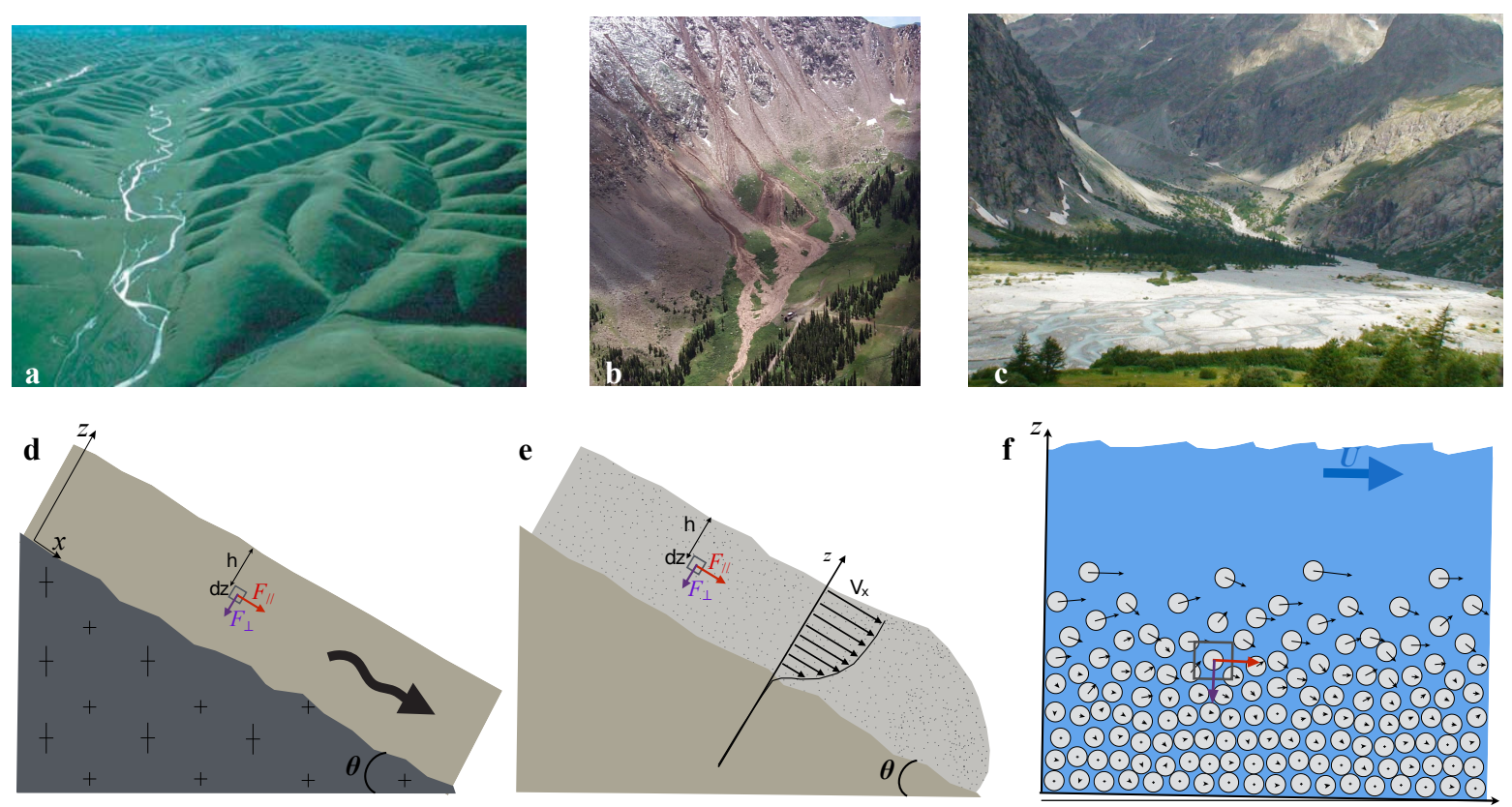

Figure 2: Pictures and sketches of the three main sediment transport systems. a) (from Perron et al. (2009), California.) and d): soil creep, mostly driven by gravity, $F_{/ /}=F_{g}+F_{s} \simeq F_{g}$. b) (Peru, credit K. Clark) and e): debris flows, dry or wet, driven simultaneously by gravity and surrounding flow shear stress, $F_{/ /}=F_{g}+F_{s}$. c) (French Alps, credit M. Houssais) and f): sediment entrainment in a river channel, mostly driven by surrounding flow shear stress, $F_{/ /}=F_{g}+F_{s} \simeq F_{s}$. The red and purple arrows represent the mean shear stress and particle pressure, respectively, applied on a unit volume in the system. 
jamming framework to describe soft matter flow ignition, $\tau_{c}$ plays the same role as the critical packing fraction $\Phi_{c}$ for confined granular systems, or the critical temperature for glasses (Liu and Nagel (2010), and references therein). Such transitions are a consequence of the disordered packing structure of the constituent particles; the inability to organize into an ordered crystalline structure means that there is not a well-defined minimum energy state. This is different from classical phase transitions such as freezing of water, in which viscosity jumps discontinuously as a step function at a well-defined critical temperature. As a consequence, while a yield criterion for flow of granular materials exists, it exists as a singularity where particle motion becomes infinitely slow. Moreover, it has been shown that as a granular system approaches its jamming point from above, the particle motion becomes increasingly correlated and progressively slower (Keys et al., 2007). In practice, this means that the transition from static to flowing grains is continuous, and the apparent threshold will be very sensitive to how it is measured. Our recent experiments revealed that this general behavior is observed for sediment transport approaching its critical shear stress $\tau \rightarrow \tau_{c}$, in terms of the diverging time scale (Houssais et al., 2015). Similarly, it is well known that averaging sediment transport data becomes more and more difficult as the stress $\tau_{x z}$ approaches $\tau_{c}$ (Recking et al., 2012).

Finally, we present here the concept of a metastable glassy state, which has been introduced to model the diversity of behaviors that arise in glass cooling. In particular, the final density of a glass - or level of disorder - at the jamming point is known to change with the rate of cooling (Mari et al. 2009). As a consequence, the jamming point of the glass is not necessarily a fixed temperature, but rather may be a function of the history of cooling. Although this idea first emerged for thermal systems, a similar modeling approach is being developed to understand the shear thickening of granular suspensions (Mari et al., 2014, Wyart and Cates, 2014). These results suggest that the critical packing fraction or shear stress for a granular system may well depend on the shear rate history. We will return to this point later in discussing evidence for history-dependence in sediment transport systems.

\section{$2.2 \quad$ Dry granular flows}

We first consider dense granular flows in the absence of a liquid, in which case $F_{s}$ results entirely from granular contact forces (no hydrodynamic forces). The local frictional rheology begins with two key assumptions: (1) there exists a well-defined yield stress associated with static friction coefficient $\mu_{s}$, below which the granular media behaves as a rigid body; and (2) the granular flow behaves locally as an incompressible fluid (Jop et al., 2005, 2006, MiDi, 2004). For simplicity we consider the $x-z$ plane of an infinitely large 3D flow. The shear stress is then $\tau=\tau_{x z}$ and the granular shear rate $\dot{\gamma}=\dot{\gamma}_{x z}$. The two relate to each other via the constitutive relation $\tau=\eta_{\text {eff }} \dot{\gamma}$, where $\eta_{\text {eff }}$ is an effective viscosity which varies as a nonlinear function of the local shear rate $(\dot{\gamma})$ and confining pressure $P_{p}(\mathrm{MiDi}, 2004)$. Because of this dependence with the pressure due to friction, it is more natural to consider local rheology in terms of an effective friction coefficient defined as:

$$
\mu \equiv \tau / P_{p} .
$$

From a phenomenological perspective, it has been proposed that the effective friction results from a competition between two timescales: a macroscopic deformation timescale associated with granular shear, $t_{\text {macro }} \equiv 1 / \dot{\gamma}$, and a microscopic inertial timescale $t_{\text {micro }} \equiv \sqrt{d^{2} \rho_{p} / P_{\perp}}$ related to particle rearrangement under a confining pressure $P_{\perp}$, where $d$ and $\rho_{p}$ are particle diameter and density, respectively (Forterre and Pouliquen, 2008, Jop et al., 2005, 2006, MiDi, 2004). The ratio of these timescales defines what has been termed the Inertial number, $I=$ $t_{\text {micro }} / t_{\text {macro. }}$. Dry granular flows (such as heap flows down inclined planes and collapsing granular columns (Jop et al., 2005, Lagrée et al., 2011)) have been shown to follow a single 
rheology:

$$
\mu(I)=\mu_{s}+\left(\mu_{d}-\mu_{s}\right) /\left(I_{0} / I+1\right),
$$

where $I_{0}$ is a constant and $\mu_{d}$ is the limiting dynamic friction at high values for $I$. Typical values used for dry granular flow experiments are $I_{0} \simeq 0.3, \mu_{s} \simeq 0.32$ and $\mu_{d} \simeq 0.7$ (Jop et al., 2005, MiDi, 2004). Importantly, since the slope is used to compute the pressure, all these concepts and results are valid for any slope $\theta>0$ ((Jop et al., 2005, Lagrée et al., 2011) $)$. This rheology may be extended to submerged dense-granular flows, where it has been shown that the confining pressure term depends on the ratio of particle to fluid densities $\rho_{p} / \rho$ and the Stokes number $S t$ (Cassar et al., 2005, du Pont et al., 2003), with $\rho$ the fluid density.

\subsection{Submerged Granular Flows}

Following the success of the dry granular rheology (eq. 2), a similar approach has been taken to model the rheology of dense suspensions of particles in a viscous fluid (viscosity, $\eta_{f}$ ), in the limit where granular collisions are viscously damped $(S t<1)$. The macroscopic timescale remains as in the dry case; however, $t_{\text {micro }}$ becomes driven principally by viscous drag. Dimensional analysis leads to:

$$
t_{\text {micro }}=\frac{\eta_{f}}{P_{p}}
$$

and the resulting timescale ratio was termed the Viscous number (Cassar et al., 2005, du Pont et al., 2003):

$$
I_{v}=\frac{\eta_{f}|\dot{\gamma}|}{P_{p}} .
$$

In the suspension case, the shear stress $\tau$ is carried by fluid and particle motion: $\tau=\tau_{p}+\tau_{f}$. Accordingly, the effective friction coefficient $\mu$ results from the sum of the particle-particle and particle-fluid interactions.

\subsection{Unification of granular flows and viscous suspensions}

It was recently proposed by Boyer et al. (2011) that the entire range of granular flows could be modeled as a smooth transition from the dry and dense case to the fully suspended condition (where granular contact forces are negligible). To examine this idea they performed experiments on neutrally-buoyant particles $\left(\rho_{p} / \rho=1\right)$ suspended in a viscous fluid, and sheared at a controlled rate in a Couette cell. We note here that this is similar to the model system originally considered by Bagnold (1954) in his foundational paper on granular rheology. The confining pressure $\left(P_{p}\right)$ was externally imposed by a plate, and the initial packing fraction $(\Phi)$ was varied independently. With this setup, Boyer et al. (2011) were able to assess the effective friction and also the packing fraction as function of the viscous number. To explain the data, they constructed a local rheology equation in which the two separate friction terms considered previously, solid contact and hydrodynamic, are added together:

$$
\mu\left(I_{v}\right)=\mu^{d r y}+\mu^{s u s p}=\mu_{s}+\frac{\mu_{d}-\mu_{s}}{1+I_{v, 0} / I_{v}}+I_{v}+\frac{5}{2} \Phi_{c} I_{v}^{1 / 2},
$$

where $I_{v, 0}=0.005$, and $\Phi_{c}=0.585$ is the packing fraction at which the effective viscosity $\eta_{e f f}$ appears to diverge in Boyer et al. (2011) experiments. The associated relationship of the bulk packing fraction $\Phi$ with the Viscous number was found to be:

$$
\Phi=\frac{\Phi_{c}}{1+I_{v}^{1 / 2}} .
$$




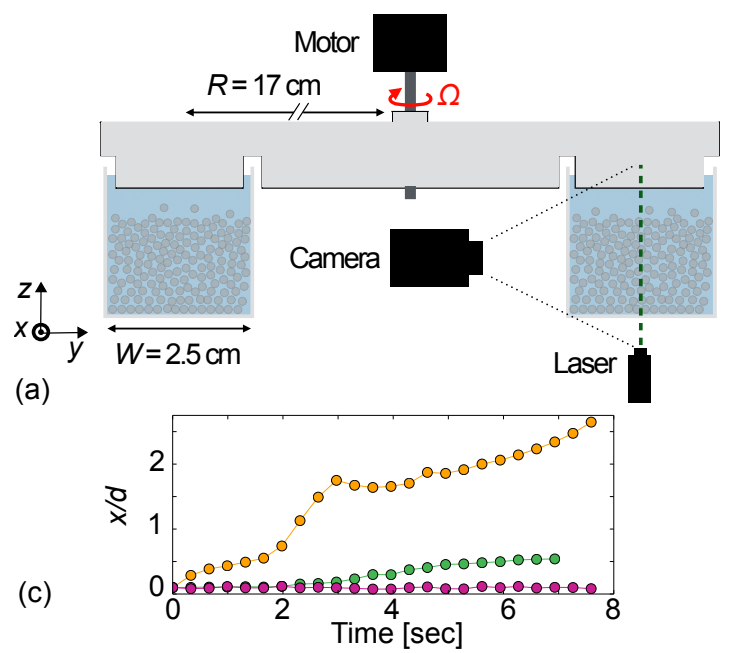

(b)

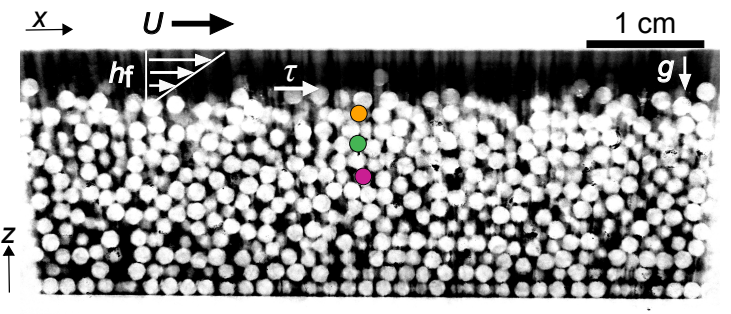

(d)

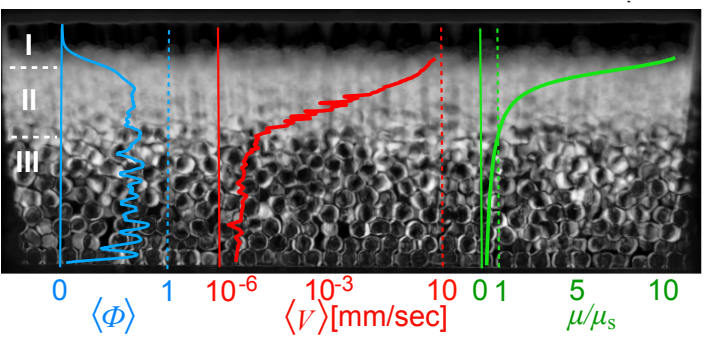

Figure 3: a) Experimental setup sketch. b) Example of sediment bed image. Yellow, green and magenta circles represent the initial positions of three particles, whose horizontal displacements are reported in panel c), during an experiment performed at $\tau^{*}=0.42$ (From Houssais et al. (2015)). d) Map of the standard deviation of pixel intensity measured over thousands of images and a duration of $10 \mathrm{hr}$, during the same experiment. The blue and red curves represent, respectively, vertical profiles of packing fraction and particle mean velocity. The green curve represents the vertical profile of $\mu / \mu_{s}=\tau /\left(P_{p} \mu_{s}\right)$, with $\mu_{s}=0.32$.

Note that, for the very dilute suspension case $\left(I_{v} \rightarrow+\infty\right)$, expressions (5) and (6) converge toward the classical Einstein (1905) viscous suspension rheology, $\eta_{e f f}=\eta_{f} \frac{\mu}{I_{v}}=\eta_{f}(1+5 / 2 \Phi)$. Conversely, for $I_{v} \rightarrow 0, \mu\left(I_{v}\right)$ approaches the value for static friction (see model curves figure 5). Equation 5 was found to describe Boyer et al. (2011) experimental results remarkably well.

There are three aspects of sediment transport that differ from these experiments: (1) particles settle $\left(\rho_{p} / \rho>1\right),(2)$ there is no confining pressure imposed on the granular bed, and (3) grains creep under conditions below the apparent yield stress as defined from a static friction (Nguyen et al., 2011). While the Boyer et al. (2011) model predicts convergence toward a static friction as the viscous number vanishes, observations of creep in other experiments suggest that the simple yield condition limit in the local rheology model may not be the complete picture. We return to this idea below.

\section{Lessons from Sediment Transport by a Laminar Flow}

\subsection{Experimental approach}

River sediment transport represents a fascinating and challenging granular flow system, in that all three granular phases may coexist; quasi-static or jammed, dense-granular flow and dilute suspension correspond to sub-threshold, bed-load and suspended-sediment transport, respectively (Durán et al., 2012, Frey and Church, 2009, 2011, Ouriemi et al., 2009). While decades of research have led to major advances in our understanding of fluid turbulence and its influence on particle entrainment and transport (Diplas et al., 2008, Schmeeckle, 2014a), the granular physics of sediment transport have received scant attention. Considering bed load transport, it is apparent that entrainment of grains at the surface of a river bed will be strongly controlled by hydrodynamic forces. However, once in motion a bed-load particle will apply a shear stress to the grains below, leading to movement below the surface layer. Although 
granular shear is explicitly considered in models for debris flows, it is rarely considered for fluvial transport (Aussillous et al., 2013, Capart and Fraccarollo, 2011, Durán et al., 2012, Frey and Church, 2011, Houssais et al., 2015). Many studies track particle motion of the surface layer of grains in bed load (Lajeunesse et al., 2010, Roseberry et al., 2012), but few observe granular dynamics deep into the bed (Aussillous et al., 2013, Revil-Baudard et al., 2015).

We have begun to explore the granular physics of fluid-driven sediment transport by examining the motion of spherical particles driven by a laminar shear flow in a small annular flume (Houssais et al., 2015, 2016), following on the work of Charru et al. (2004) and Aussillous et al. (2013). The confounding effects of fluid turbulence are intentionally suppressed by maintaining low Reynolds numbers $\left(R e \sim 10^{0}\right)$, which also means that collisions are damped $(S t<1)$, bringing granular-friction effects to the fore. Although simplified, it has been demonstrated that sediment transport under these conditions shows many similarities to the more complicated case of turbulent flows and heterogeneous particle sizes and shapes (Charru et al., 2004, Houssais and Lajeunesse, 2012, Malverti et al., 2008). Details of our experiment and results have already been presented (Houssais et al., 2015, 2016). Here we outline our approach and highlight pertinent findings that serve to motivate the rest of the paper. The experimental apparatus is a small annular flume (see Fig. 3) with smooth walls and bottom, which allows us to approximate an infinitely long and deep river channel. The fluid and sediment used were, respectively, viscous oil $\left(\eta_{f}=68.6 \eta_{\text {water }}\right)$ and light $\left(\rho_{p}=1.13 \rho\right)$ PMMA particles of diameter $d=1.5 \mathrm{~mm}$. We matched the refractive index of the oil with that of the particles, minimizing light attenuation in the granular pack. We then impregnated the oil with a dye that was fluoresced with a laser sheet; this refractive index matched scanning (Dijksman et al., 2012) technique allows one to do image-based tomography of the granular bed. Here we report observations from an $x-z$ plane located at the center of the channel (see figure $3 \mathrm{a}$ and $\mathrm{b}$ ). We tracked all particles (see trajectories example figure 35) and measured packing fraction and velocities over thousands of images recorded over durations ranging from $10 \mathrm{hr}$ to $30 \mathrm{hr}$. A rotating top plate shears the system at a constant mid-channel velocity $U$. After an initial period of bed compaction, the fluid flow depth $h_{f}$ saturates to a constant value, allowing us to estimate the flow boundary shear stress, $\tau=\eta_{f}\left(U-\left.\langle V\rangle\right|_{z=z_{s}}\right) / h_{f}$, with $\left.\langle V\rangle\right|_{z=z_{s}}$ the average particle velocity at the bed surface elevation $z_{s}$. We then compute the classical dimensionless Shields number:

$$
\tau^{*}=\frac{\tau}{\left(\rho_{p}-\rho\right) g d} .
$$

\subsection{Phenomenology}

It is important to point out that - in contrast to many granular rheology couette-cell experiments - the sediment bed has a free-surface condition, so there is not an imposed confinement pressure due to the presence of a plate. This means that the confinement pressure is due only to the hydrostatic overburden of the grains themselves. As a consequence, the confinement pressure $P_{p}$ varies vertically as a function of the packing fraction profile $\Phi(z)$ which may be written (Houssais et al., 2015, 2016)

$$
P_{p}(z)=\left(\rho_{p}-\rho\right) g \cos \theta \int_{z}^{\infty}\langle\Phi\rangle(z) d z=\left(\rho_{p}-\rho\right) g \cos \theta \Sigma(z)+P_{0}
$$

where $\Sigma(z)=\sum_{z}^{\infty}\langle\Phi\rangle(z) d z$ and $P_{0}$ is a constant of integration. Note that in our experiment, as $\theta=0, \cos \theta=1$. Physically, the value for $P_{0}$ corresponds to the normal stress on a particle at elevations where $\langle\Phi\rangle$ approaches zero; it is related to the weight of an individual particle. We have proposed (Houssais et al. 2016) that $P_{0}$ connects the local flow rheology to the classic 


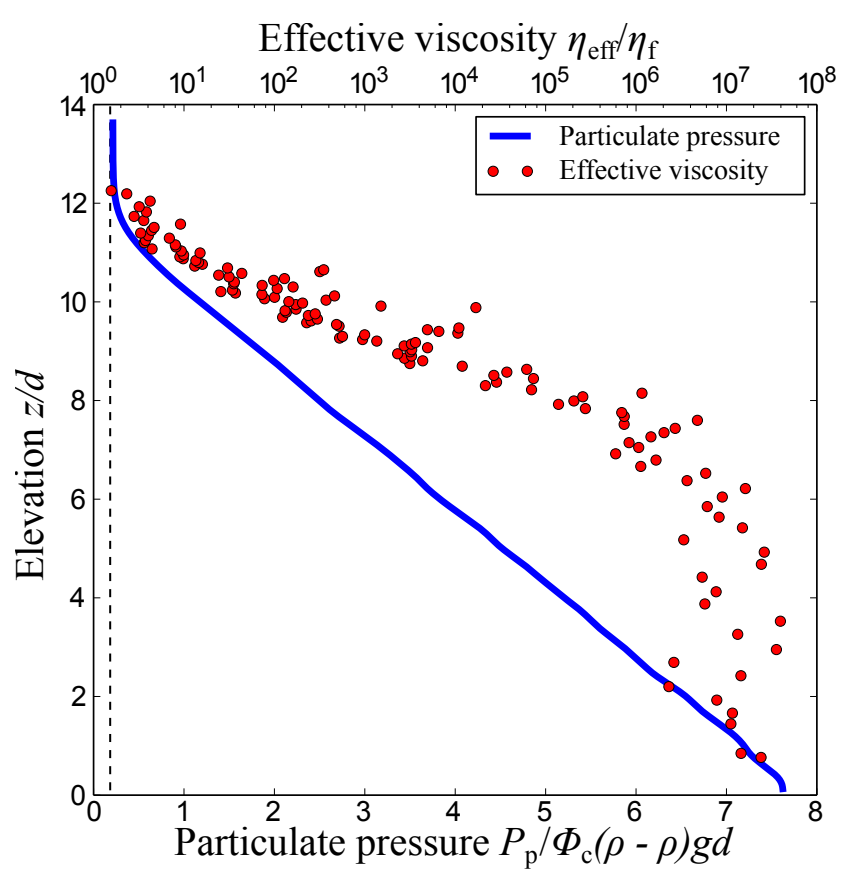

Figure 4: Vertical profiles of the normalized particle pressure $P_{p} /\left(\Phi_{c}\left(\rho_{p}-\rho\right) g d\right)$ (from equations 8 and $9 p$ and effective viscosity $\eta_{e f f} / \eta_{f}$, for $\tau^{*}=0.42$. The effective viscosity comes directly from the measured velocity profile: $\eta_{\text {eff }}=\tau / \dot{\gamma}=\tau /(d\langle V\rangle / d z)$. The dashed line indicates the asymptotic case of no particles $(\Phi \rightarrow 0)$, where $P_{p} \rightarrow P_{0}$ and $\eta_{e f f} \rightarrow \eta_{f}$.

definition of the critical stress at the bed surface for entrainment of a static object (Shields 1936, Wiberg and Smith, 1987):

$$
P_{0}=\frac{\tau_{c}}{\mu_{s}},
$$

with $\mu_{s}=0.32$ found by Boyer et al. (2011), and $\tau_{c}=0.08 \mathrm{~Pa}$ inferred for our system, which corresponds to a critical Shields number $\tau_{c}^{*}=0.04$. Although this value is small compared to those reported in some other laminar bed-load experiments, it is similar to the value previously found for a loose bed - before compaction - in experiments conducted by Charru et al. (2004) under experimental conditions similar to ours (Houssais et al., 2016).

For five different Shields numbers (0.12 to 0.42), we computed the vertical profiles of timeaverage packing fraction $\langle\Phi\rangle(z)$ and particle streamwise velocity $\langle V\rangle(z)$. Profiles obtained for the experiment at $\tau^{*}=0.42$ are shown in figure $3 \mathrm{~d}$, where the background is the standard deviation of images recorded over $10 \mathrm{hr}$ of steady-state sediment transport. The latter demarcates an upper regime where grains have experienced significant motion over the time period observed, and a lower regime with much slower and spatially variable motion. The first thing to notice is the huge range of mean particle velocity values resolved - from $\langle V\rangle \sim 10^{-2} \mathrm{~m} / \mathrm{s}$ at the surface to $\langle V\rangle \sim 10^{-9} \mathrm{~m} / \mathrm{s}$ at the bottom of the channel - which encompasses much of the range of particle velocity relevant for natural sediment transport, from hillslope creep to bed load. Considering the particle concentration profile, it increases rapidly across the fluid-sediment interface, and then remains more-or-less constant with depth below the surface. Figure 4 shows the vertical profiles of the normalized particle pressure and effective viscosity for the experiment made at $\tau^{*}=0.42$. Notice the sharp increase of viscosity, $\eta_{e f f}=\tau /(d\langle V\rangle / d z)$, over the few first particle diameters below the surface, plateauing at a value of $10^{7} \eta_{f} \sim 10^{6} \mathrm{~Pa}$.s. For comparison, this viscosity is significantly higher than a lava flow $\left(\eta_{\text {eff }} \sim 10^{3}\right.$ Pa.s $)$, but significantly lower 
than a glacier flow $\left(\eta_{\text {eff }} \sim 10^{13} \mathrm{~Pa} . \mathrm{s}\right)$.

From our experimental measures of $\langle\Phi\rangle(z)$ and $\langle V\rangle(z)$, we can qualitatively identify three regimes of fluid-driven sediment transport (Houssais et al. 2015$)$. Regime (I) is a dilute suspension characterized by sufficiently low sediment concentration such that grain-grain friction is negligible. Below this is regime (II), where packing is dense $(\Phi>0.2)$ and the velocity profile decays exponentially with depth below the surface, in agreement with the few previous studies (Aussillous et al., 2013, Capart and Fraccarollo, 2011). This corresponds to a dense-granular flow regime that we associate with bed-load transport. The bottom of Regime (II) is demarcated by a kink in the velocity profile (at $z \simeq 7 d$ in the example shown Fig. 3 3 ) beyond which the velocity decays with depth at a much slower rate. In terms of rheology, a kink is observed in the effective viscosity profile (Fig. 4). Below this kink is Regime (III), which had not been previously reported in sediment transport experiments as earlier studies did not record such slow velocities. This zone of very slow velocity (and velocity decay) corresponds to observed dry granular creep, in which a quasi-static bed undergoes intermittent rearrangement (Nguyen et al., 2011, Nichol et al., 2010).

\subsection{Unified rheology for bed load and suspension}

To deepen our understanding of the coexistence of these 3 observed regimes, we confront our data with the granular rheology presented in section 2. In particular, we consider the ability of the local rheology model of Boyer et al. (2011) to describe the dynamics of sediment transport across all regimes. The reader is referred to Houssais et al. (2016) for a full exposition of the results and their derivation. From our experimental profile of particulate pressure and shear rate (see example Fig. 4), we compute and plot a profile of the local friction as a function of depth; doing so reveals that friction decreases nonlinearly but monotonically (Fig. 3d). Intriguingly, the inferred depth associated with the transition to creep coincides with the point at which the local friction is equal to the static value. Further, it appears that the local friction actually decreases below the static friction value in the creep regime (III), as observed previously in recent numerical simulations of dry granular flow (Koval et al., 2009, Singh et al., 2015).

In order to test the $\mu\left(I_{v}\right)$ rheology model (eq. 5), we computed time-averaged values of $\mu$ and $I_{v}$ at all depths for each experiment at different Shields number $\tau^{*}$. We observe a remarkable collapse of the data onto a single $\mu\left(I_{v}\right)$ curve for values of $10^{-5} \leq I_{v} \leq 1$, and these data are very well described by the Boyer et al. (2011) model (see Fig. 5a). In the limit of very large values of $I_{v}$ - which corresponds to the most dilute part of the flow $(\Phi \rightarrow 0$ and $\left.P_{p} \rightarrow P_{0}\right)$ - individual grains interact only with the fluid and the confining pressure is only the weight of a particle. We expect the friction coefficient of these particles to approach a limiting value associated with dynamic friction of a single grain, i.e., $\mu=\tau / P_{0}$, instead of following the suspension curve indefinitely; the expected limits are indicated for each Shields number in figure 5 a (color dashes on the right axis). There are two critical values for $I_{v}$ that correspond to changes in the transport regimes previously identified. The value $I_{g s}=0.2$ marks the point where the dry granular and suspension rheology curves intersect $\left(\mu^{d r y}=\mu^{\text {susp }}\right)$, and coincides with the identified transition point between regime (I) and regime (II). The second critical value $I_{c g}=10^{-5}$ occurs where the friction coefficient is equal to the static value, and marks the transition from bed load to creep.

Thus, the rheology of sediment transport across bed-load and suspension regimes is completely described by a single constitutive relation, eq. (5), which combines the limiting dynamics of a dense granular flow and a dilute suspension. In that rheological framework, we redefine bed load as regime (II), and suspension as regime (III). Such a result was anticipated by several recent theoretical studies (Chiodi et al., 2014, Ouriemi et al., 2009, Revil-Baudard and Chauchat, 

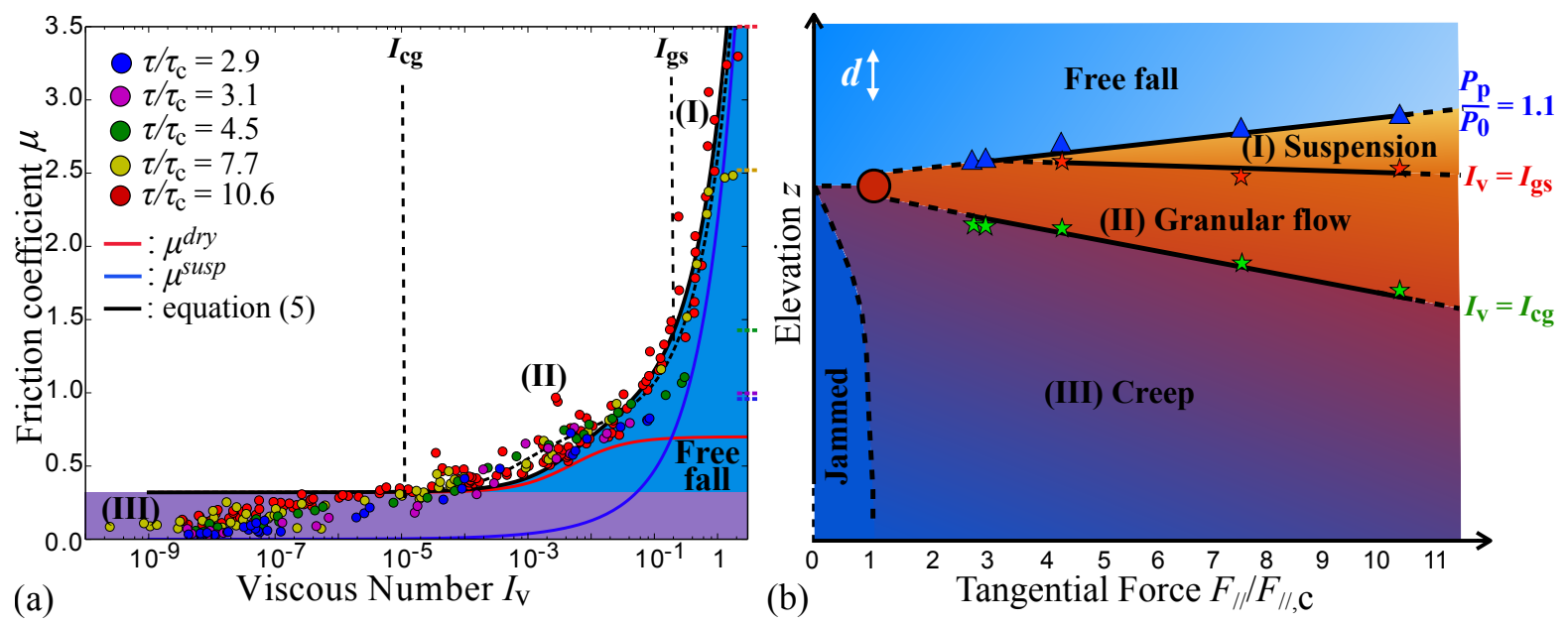

Figure 5: a) Sediment transport rheology. Friction coefficient $\mu$ as a function of the Viscous number $I_{\mathrm{v}}$, for five different Shields numbers in the vicinity of the critical Shields number $\tau_{\mathrm{c}}^{*}=0.04$. The red and blue curves represent $\mu^{d r y}\left(I_{\mathrm{v}}\right)$ and $\mu^{\text {susp }}\left(I_{\mathrm{v}}\right)$ respectively, and the solid black curve represents equation (5) with values adopted from Boyer et al. (2011). The black dash curve is a best fit result using equation (5) where $\mu_{\mathrm{s}}$ is the only fixed parameter. Vertical black dashed lines marks regime transitions: at $I_{v}=I_{c g}=10^{-5}$, from creep (III) - defined by $\mu \leq \mu_{\mathrm{s}}$ (the magenta area) - to dense-granular flow (II); and $I_{\mathrm{v}}=I_{\mathrm{gs}}=0.2$, from densegranular (II) to suspension flow (I) which, by definition, corresponds to the intersection of the red and blue curves. The dashed color lines on the right side indicate the respective asymptotic values $\mu \rightarrow \tau / P_{0}$ for each experiment. The end of the data curves correspond to the very dilute $(\Phi \rightarrow 0)$ part of the flow, which may be considered the free fall regime where we expect convergence toward $\tau / P_{0}$ (blue area). b) Proposed regime diagram of fluid-sheared granular transport based on our experiments, where the $x$ axis is the tangential force normalized by its critical value for particle entrainment at the bed surface $F_{/ /} / F_{/ /, c}$. For our experimental results, as $\theta=0$, the gravity component to the tangential force is null $F_{g}=0$, then: $F_{/ /} / F_{/ /, c}=\tau / \tau_{c}$ (axis made with $\tau_{\mathrm{c}}^{*}=0.04$ ). The $z$ axis is the elevation with an arbitrary origin, and with scale of grain size $d$ indicated. The red dot marks the bifurcation of regimes (II) and (III) that occurs when the onset of bed-load transport intersects the surface. The Boyer et al. (2011) rheology model describes regimes (I) and (II), but not (III). There must be a sufficiently low stress for which all motion ceases and the granular bed is jammed (hypothetical area marked "Jammed" on the diagram). All hypothetical regions are denoted by dashed lines; solid lines are delineated from our data but are meant only to guide the eye. 
2013).

From this fundamental result, we present in figure $5 \mathrm{~b}$ an updated version of the regime diagram for liquid-driven sediment transport that we proposed earlier (Houssais et al., 2015). On this figure, we report our experimental observations of the regime transition positions, as a function of the normalized applied tangential force $F_{/ / /} / F_{/ /, c}$ (strictly equivalent to $\tau / \tau_{c}$ in our configuration where $\theta=0$ ). Naturally we plot the positions of the two critical Viscous numbers defined above, and we also report the elevation at which the pressure drops bellow $1.1 P_{0}$, as an arbitrary assessment of the transition to the Free Fall regime (to provide a reliable elevation measurement assessing where $P_{p} \rightarrow P_{0}$ ). Three major implications emerge from this diagram. First, the regime transitions all shift vertically with changing shear stress; in particular, if we consider a "total active layer" delimited by the the limit $I_{v}=I_{c g}$ and $P_{p}=1.1 P_{0}$, it is clearly thickening with the stress. The result is similar if we consider only the granular flow (or newly defined bed load) layer or the suspension layer. Second, the transition to free fall and the transition to creep appear to meet for $\tau=\tau_{c}$, which reinforces the notion that the inferred $P_{0}$ value corresponds to the critical stress for particle entrainment at the bed surface. Third, the transition to suspension appears to start at $\tau \simeq 5 \tau_{c}$, a value which was reported from many other sediment transport studies (often driven by a turbulent flow), without clear physical basis. Here this transition emerges naturally from the rheology, and the evolution of the free surface of the granular bed.

\subsection{Linking rheology to flux}

Unifying the rheology of bed-load and suspension opens the possibility of modeling the continuum of fluvial transport with a single law. In terms of sediment flux, it can be cast as the integrated product of the sediment concentration and velocity profiles. Predicting sediment flux using the rheology framework requires knowledge of the following elements: the fluid boundary shear stress $\tau$, and the particle size distribution and density which are used to assess $P_{0}$. One can then estimate the maximum values for the Viscous number and the friction coefficient $\mu\left(I_{v}\right)=\tau / P_{0}$, and use them to extrapolate the $\langle V\rangle$ and $\langle\Phi\rangle$ profiles from stream surface measurements. Consequently, one can compute the local total sediment flux as:

$$
\left\langle q_{s}\right\rangle=\rho_{p} \int_{0}^{+\infty}\langle V\rangle \cdot\langle\Phi\rangle d z .
$$

\subsection{Creep and breakdown of rheology}

Perhaps more surprising even than the remarkable success of $\mu\left(I_{v}\right)$ rheology for bed load and suspension, is the perplexing dynamics associated with creep. Based on their experimental results and theoretical development, Boyer et al. (2011) sensibly predicted that $\mu \rightarrow \mu_{s}$ as $I_{v} \rightarrow 0$; in other words, that friction converges to the static value at vanishing shear rate. This prediction is in line with the idea that the granular bed has a yield criterion associated with static friction, and is consistent with the jamming transition observed for confined systems. Instead, with data that extend the lower range of Viscous number by several orders of magnitude, we observe that friction continues to decrease below the static value with decreasing $I_{v}$. Rather than $\mu=\mu_{s}$ and $I_{v}=I_{c g}$ corresponding to a transition from flow to no motion, it marks a transition from granular flow to creep. It is currently a fundamental question whether the free-surface condition of our experiments and most natural systems - as opposed to confined geometries of rheology studies - somehow facilitates creep instead of jamming. We know of no other granular experimental studies in which the rheology of creep has been directly quantified, so we cannot compare our findings to others. However, previous dry granular experiments have 
reported indirect observations of creeping motion below the yield criterion (Nguyen et al., 2011. Nichol et al., 2010), and this is a rather general behavior that is expected for fragile granular solids (Jaeger et al., 1996). This comparison suggests that creep observed in our experiments may be similar to dry granular creep, and hence related to hillsope soil transport. In addition, bulk material creep has been observed in soil mechanics studies (Mitchell, 1993, Vyalov, 1986) and in non-Newtonian fluids (Barnes, 1999). Therefore, while at first confusing, observation of creep is consistent with our sub-static friction coefficient values. While our experiments used smooth spherical particles in an idealized setting, we believe the findings to be general. Previous work has reported creep in a variety of particle shapes (Komatsu et al., 2001, Vyalov, 1986); so while particle shape and size may influence the magnitude of creep, it doesn't appear that making particles non-spherical acts to shut off the creep mechanism. A final point worth making is that the data do not collapse onto a single curve in the creep regime. Instead, for $I_{v}<10^{-5}$ (i.e., creep) we observe a systematic effect of the driving shear stress on friction, which is not seen outside of the creep regime (Fig. 5). These results indicate, at the very least, that the local rheology model is incomplete and that some additional parameters or relations are needed to understand measured friction.

The proposed regime diagram (Fig. 5b) is based on a limited and specialized experimental set, and it is not known to what extent Earth-material flows may be unified under such a framework. We are optimistic about its applicability, however, since the behavior of fluiddriven sediment transport is dynamically similar to dry granular flows and viscous suspensions. Below we speculate how Earth-material flows and their transitions could be mapped to this framework.

\section{Linking Granular Physics with Landscape Dynamics}

\subsection{Ubiquity of creep (III) in the environment}

Our finding of creep behavior below the apparent threshold of motion, and deep below the surface of the granular bed, goes against a main hypothesis used to apply granular physics to landscape dynamics: sediment flow vanishes at the jamming point, and that bed load occurs as a thin layer of grains flowing over a static pack. This hypothesis has been used to solve particle and fluid flow equations in recent studies (Chiodi et al., 2014, Durán et al., 2012, Ouriemi et al., 2009), but it appears from our experiments that the bottom boundary condition of granular flows - in particular when one is interested in the long-time system evolution — should be investigated more carefully. If creep is ubiquitous it presents new theoretical challenges, but also opens exciting new perspectives for geomorphology.

\subsubsection{Investigating soil creep again}

In landscape dynamics, creep has been invoked as a relevant transport process only for the case of "diffusive" soil transport down hillslopes. On hillslopes, creep is manifest as the slow (i.e. with negligible inertia), gravitationally-driven downslope transport of grains on slopes below the angle of repose. In order to explain how flow can occur below the apparent yield stress of the soil, researchers point to continual disturbance by physical and biological agents (rainsplash, freeze/thaw, burrowing, tree throw, etc.) as causing dilation of the soil (see Anderson and Anderson (2010) for a nice summary). This dilation, in turn, is supposed to reduce the local friction of the soil and essentially lower its yield stress. In the laboratory, disturbance-driven creep has been simulated by perturbing sand acoustically (Roering et al., 2001). Those experiments, however, gave a diffusion coefficient that was three orders of magnitude larger than values inferred from natural systems. Moreover, our experimental results show that creep can 
also occur as a consequence of an applied shear stress, in the absence of any external sources of bulk dilation. Importantly, we have also observed creep in the absence of a bed load layer, for an applied fluid shear stress that was at the critical value for granular flow initiation: $\tau^{*}=0.04$ (see movie 3 in supplementary informations of (Houssais et al., 2015)).

Considering these results, and additional findings from dry granular systems (Nguyen et al. 2011, Nichol et al., 2010, Pons et al., 2015, Reddy et al., 2011), it seems likely that creep occurs in soils for tangential forces lower than the critical $F_{/ /}<F_{/ /, c}$ - meaning for slopes lower than the angle of repose $\theta<\theta_{c}$ - and that it may occur even in the absence of an external source of disturbance. Particle size, shape and surface property, and external perturbations such as fluid flow or biological agents, may certainly influence granular creep in nature. However, it is possible that these factors influence the rate of creep while leaving the underlying mechanism the same. The dynamics of creep are so slow that field studies linking granular motion to bulk flow, in hillslopes or rivers, are exceedingly difficult. A focused effort on examining the rheology of creep in fluid-driven and dry granular systems in laboratory experiments is warranted. Field efforts could work to produce better resolved soil creep velocity profiles on hillslopes. Analysing monitoring data of slow landslides also appears to be a promising approach in that direction (Di Maio et al., 2013).

\subsubsection{Consequences of creep: aging and hysteresis}

The creeping regime is the least understood state of granular transport as it is not described by the $\mu\left(I_{v}\right)$ rheology. Although quite slow, its persistence and spatial extent on soil-mantled hillslopes means that creep is a major contributor to landscape erosion and evolution. If creep also occurs in rivers, it is likely a minor contributor to the overall sediment flux compared to bed and suspended load. However, creep is known to drive slow and persistent compaction of a loose granular bed (Knight et al., 1995) over time. Granular flows generally drive the opposite, i.e., dilation of the bed (visible on figure 5, where the line define by $P_{p}=1.1 P_{0}$ is going up with shear). Because packing fraction is related to particle contact and hence friction, the net effect is that the apparent yield stress of the granular material will change through time as a function of shear. The consequence is that the yield stress of a granular material is a function of the history of applied stress or shear rate, a phenomenon reffered to as aging and rejuvenation (Pons et al., 2015). These effects, however, have barely garnered mention in geomorphology. It has been suggested that bed compaction resulting from bed-load transport could act to increase the threshold entrainment stress through time, as particles arrange themselves into a more tightly packed bed state (Charru et al., 2004, Turowski et al., 2011) — a result that has been qualitatively confirmed in our experiments and that awaits further analysis. The important point here is that creep alone in rivers may exert a strong influence on the sediment transport rate, by increasing the critical Shields number through time as the granular structure of the river bed rearranges to a more stable state. On the other hand, large floods that cause vigorous transport could act in the opposite direction by dilating the bed, and destroying the granular fabric. These dynamics may contribute significantly to observations of bed-load flux hysteresis in rivers (Hsu et al., 2011, Jerolmack, 2011).

\subsection{Failure and onset of granular flows: transitions out of regime III}

\subsubsection{Granular dynamics of flow initiation}

Many researchers have examined the collapse of a granular pile, in which grains are suddenly released by lifting of a gate or wall, and flow by gravity until they come to rest. The simplest experiments involve dry grains initially prepared by sedimentation in air (Balmforth and Kerswell, 


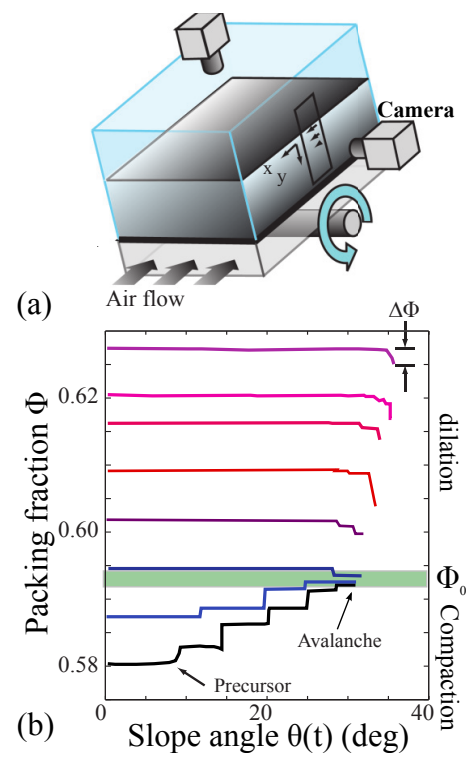

(c)

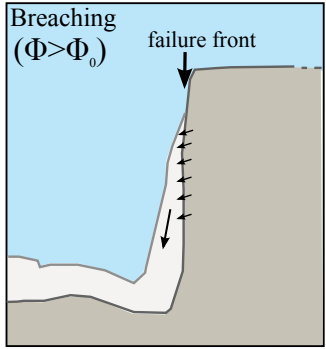

(d)

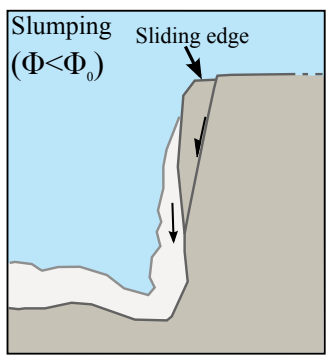

Figure 6: Transition from creep to free fall or suspension. Left: a) Experimental sketch and (b) results showing the evolution of packing fraction due to a forced, gradually-increasing slope, up to the point of failure (Avalanche) for a dry sand pile where the initial packing fraction is finely controlled (reproduced from Gravish and Goldman (2014)). Right: Sketches of the two main types of submarine slope failure - (c) breaching and (d) slumping (inspired by You et al. $(2014 \mathrm{a}))$.

2005, Lajeunesse et al. 2004, Lube et al., 2004), and result in simple scaling relations between the final runout distance and thickness of the granular pile, and its initial dimensions. Numerical simulations indicate that resulting flow dynamics from such experiments are consistent with $\mu(I)$ rheology (Lagrée et al., 2011). These studies may be considered to be the simplest model system for a landslide. More recently, studies have begun to investigate the influence of (1) a viscous ambient fluid (e.g. water or water + oil), and (2) the degree of compaction of the initial granular pile; the former is relevant for considering subaqueous mass flows, while the latter is important for all flows. These experiments have produced a startling result: unlike in air, in a viscous fluid there is no relation of the granular flow with initial dimensions of the granular pile (Pailha et al., 2008, Rondon et al., 2011). By manipulating the initial packing density with vibration, Rondon et al. (2011) revealed that this variable exerted a primary control on collapse and flow dynamics. Initially loose piles exhibited fast collapse and lower-sloping deposits, while highly compacted piles collapsed much more slowly and produced steeper deposits. The latter behavior cannot be captured by existing rheological models (Savage et al., 2014). These findings indicate that granular collapse dynamics are strongly controlled by dilation or compaction of the granular medium. This is supported by experiments conducted by Roche and colleagues in a laboratory model for pyroclastic flows: the release of gas-fluidized particles by a "dam break" failure. They found that a highly-fluidized initial pile of fine grains behaved as an inviscid Newtonian fluid for some period after release, until sufficient gas escaped to allow establishment of granular frictional contacts (Roche, 2012, Roche et al., 2004, 2008). On the other hand, coarse grains and a low degree of initial fluidization both produced granular-friction dominated flows. From the perspective of the $\mu(I)$ rheology, ambient fluid viscosity and granular packing density both exert an influence on the effective viscosity of the initial granular pile. While Roche (2012) suggests that the $\mu(I)$ framework may be relevant for modeling pyroclastic flows, the granular rheology framework has not been directly applied or tested in these experiments. 
Few granular collapse experiments have observed grain-scale motion, and so the underlying dynamics are largely unknown. A recent exception is the dry avalanche experiments of Gravish and Goldman (2014), who examined the fine details of packing fraction evolution before failure of a granular pile. These researchers tuned the initial packing fraction, then slowly increased the bed slope over time while observing granular motions in the pack. Their main observation (see Fig. 6a) was that, for initially loose (non-compacted) granular beds, there were numerous precursor events associated with localized granular rearrangement and compaction, before a "final compaction event" triggered an avalanche. As the initial packing fraction was increased the number of precursors decreased and, beyond a critical packing fraction, dilation occurred just prior to an avalanche. It is clear that there is a link between slow particle rearrangement - which is likely creep - and the triggering of an avalanche which leads to a granular flow, but the details of this link are not yet understood. From the perspective of our regime diagram, this type of failure marks a creep to dense-granular flow (III $\rightarrow$ II) transition.

\subsubsection{Triggering of landslides and turbidity currents}

In pioneering work, Iverson et al. (2000) showed that nature-scale-laboratory wet landslides behave very differently depending on the initial packing fraction. For high initial packing fractions, slope failures were associated with dilation - similar to the dry granular experiments described above (Gravish and Goldman, 2014). This dilation produces a fluid pore pressure drop (fluid moves down), which acts to stabilize the granular structure and leads to a continuous avalanche. Conversely, for low initial packing fraction the onset of failure is associated with compaction, which drives an increase in pore pressure (fluid moves up). This excess pressure destabilizes the system, and is more likely to result in slumping. Pailha and Pouliquen (2009) confirmed and formalized this dynamical feedback between slow granular and fluid motion, in a fully submerged experimental setup, and they associated the pre-failure granular motion with creep. This provides an interesting linkage between landslides and turbidity currents, in which a failing granular pile may mix with surrounding waters to create a dilute, particle-laden gravity current. In particular, the phenomeon of breaching involves the slow and continuous retreat of the front of a granular pile as grains are spalled off of the (near-)vertical face to create a turbidity current (Eke et al., 2011, Mastbergen and Van Den Berg, 2003) (Fig. 6b). Experiments demonstrate that this occurs for initially dense granular packs, in which failure is associated with dilation and negative pore pressure that acts to stabilize the face (You et al., 2012, 2014a b) - similar to the Iverson et al. (2000) observations for wet landslides. In contrast, failure of loose and muddy deposits involves compaction and the generation of positive pore pressure, and results in slumping (Mastbergen and Van Den Berg, 2003, You et al., 2012, 2014a b). While some aspects of turbidity currents and their generation appear to be similar to grain-flow failures, no studies to date have examined the granular physics associated with these flows. The eroding granular front in a breaching experiment represents a granular-shear flow, which should induce motion inward of the interface through grain-grain friction. This granular shear represents an important but unrecognized momentum sink, that ultimately must contribute to the erosion dynamics at the front.

Although our regime diagram was derived under steady conditions from a specific experiment, we believe it serves as a useful framework for considering the possible regime transitions associated with failure and flow. Experiments and field observations show that granular creep may occur for sub-threshold transport conditions, and indicate that creep also plays a critical role in the triggering of granular flows. While these dynamics are clearly associated with pore pressure changes when granular flows are wet or submerged, the similar granular dynamics observed in dry flows indicate that such effects are not essential. The failure of dry or wet 

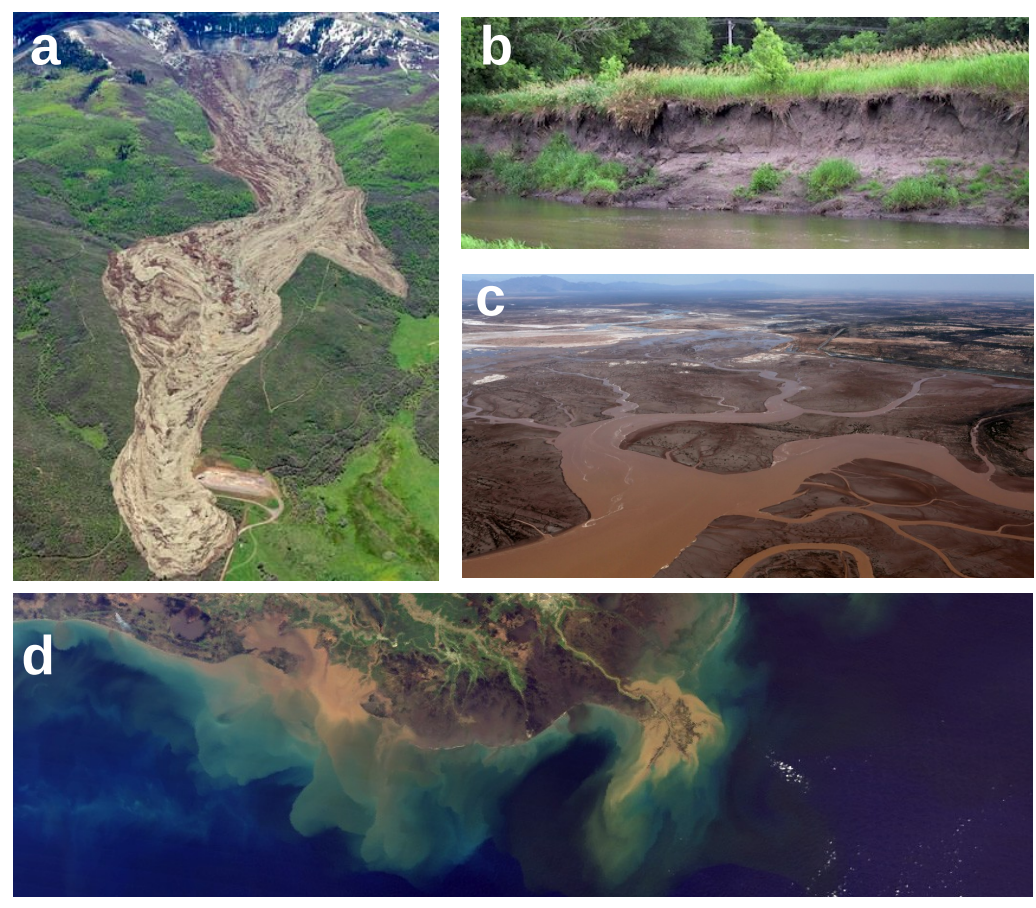

Figure 7: Examples of mud landscapes and dynamics. a) A muddy debris flow mobilized by a landslide in Colorado (Credit: John Wark, thedenverchannel.com). b) Typical muddy stream bank (Credit: Jim Colbert, Iowa State University). c) Tidal channels formed by mud deposition and erosion on the Colorado River (Credit: Francisco Zamora, Sonoran Institute, with aerial support from LightHawk). d) Plumes of mud issuing from the Mississippi and Atchafalaya Rivers during the 2011 flood; river-deposited mud comprises up to $80 \%$ by volume of the Mississippi Delta (Credit: NASA).

granular materials into avalanches, landslides and debris flows represents a regime transition from creep to dense-granular flow (III $\rightarrow$ II). Interestingly, turbidity-current breaching would appear to map to a (III $\rightarrow$ I) transition, i.e., a kind of granular sublimation. It may be that a thin granular flow regime (II) exists at the interface of the retreating front, however existing breaching experiments have not recorded detailed granular motions.

\subsubsection{Mud dynamics and landscape implications}

Predicting the onset and rate of erosion of mud is key for modeling the retreat of river banks and marshes, and bed-surface change in estuaries (see Fig. 7p). For these problems, mud is treated as a solid with a well-defined critical shear stress for entrainment. The erosion rate is then modeled as some function of the fluid stress in excess of critical. The major shortcoming of this approach is that it is entirely empirical. The critical stress is determined by a variety of methods, but they are usually related to applying a controlled fluid shear and measuring the point at which particle detachment begins (Parchure, T.M. and Mehta, A.J., 1985). The critical stress is a sensitive function of the cohesive strength of the material - which is often related to clay content - but may also vary through time (see Mehta, A.J. et al. (1989) and references therein). At some level, the effective yield strength of mud must be connected to interparticle attraction and capillary pressure, however few studies examine the particle scale. In addition, mud compacts through time with a concomitant increase in rigidity and decrease in erodibility (Mehta, A.J. et al. 1989). These changes must be related to changes in the 
granular structure of mud, however this has not been explored in any detail. Mud erosion very likely represents a granular solid to dilute suspension transition, because a large shear stress is required to overcome cohesive force and entrain grains, which go straight into suspension due to their small size. If we assume that for cohesive material the only change in our phase diagram is that the critical shear stress is larger (i.e. red dot moves to the right on the $P_{p} / P_{0}=1.1$ line), then mud erosion can be directly a regime (III $\rightarrow$ I) transition at the surface of bed (Fig. 5).

Understanding the sedimentation and compaction of mud is also a longstanding problem, especially in estuaries (Mehta, A.J. et al., 1989). Here, river currents slow and encourage deposition of silt and clay. Mixing with saline waters induces aggregation (flocculation in the geological literature) of these particles by screening of surface-charge repulsion, which further enhances sedimentation (Winterwerp, 2002). As particles sink to the bottom of the water column, however, sediment concentration increases to the point where repulsive hydrodynamic interactions cause sedimentation rates to decline significantly below the Stokes settling value. This latter effect is the well known "hindered settling" problem and is reasonably well understood for the case of non-attracting spheres (e.g., Ham and Homsy (1988)). Researchers have built upon this model to include the effects of aggregation on settling, with results that show promise when compared to experiments and field data from an estuary (Dankers and J. C. Winterwerp, 2007, Winterwerp, 2002). However, there are no direct particle observations of the sedimentation process in the presence of aggregation and shear, nor have the control of these variables been systematically explored. Moreover, while researchers have invoked fluid shear as a driver of aggregation by enhancing mixing, sufficient shear will destroy aggregates (Kranenburg, 1999). Whether and how fluid shear transitions from enhancing to suppressing aggregation, and what the influence of this is on sedimentation rates, requires further exploration.

The rheology of mud suspensions has received widespread attention, in part because of the hazard posed by destructive muddy debris flows (see Iverson (1997) and references therein; examples are illustrated Fig 7). Although mudflows have long been modeled as a Bingham fluid (Imran et al. 2001) - i.e., a Newtonian fluid for stresses above a yield stress - it is now widely recognized that they exhibit a shear-rate dependent viscosity in which the particle concentration emerges as a key controlling parameter (Ancey, 2007, Coussot, 1995, Coussot and Piau, 1994, Iverson, 1997, Iverson and George, 2014, Major and Pierson, 1992, O'Brien and Julien, 1988). The Boyer et al. (2011) local rheology formulation bears remarkable similarity to the earlier mud-suspension rheology proposed by Coussot (1995), making it a promising candidate for modeling mudflows across the dense to dilute regimes. In addition, recent studies on shear thickening in suspensions of non-Brownian hard-spheres (Mari et al., 2014, 2015, Wyart and Cates, 2014) have revealed the grain-scale connections between local sediment concentration and granular shear rate, which may eventually illuminate the particle micro-structural origins of the observed bulk rheology. However, it is known that at a critical particle concentration, mud aggregates form an interconnected network and undergo a transition to a gel (Winterwerp, 2002) - a fragile solid. We hypothesize that gelation of mud represents a transition to creep which, as discussed above, is not accommodated for in existing local rheology models. Thus, understanding the creep regime may be the key to unlocking the conditions associated with failure and freezing of mudflows. Moreover, the gelation of sedimented mud in estuaries and continental shelves is thought to be responsible for the generation of so-called "fluid muds" - concentrated and quasi-stable suspensions of mud that may suddenly destabilize and flow (Traykovski et al., 2000, Winterwerp, 2002). We hypothesize that this corresponds to a regime $(\mathrm{III} \rightarrow \mathrm{I}$ ) transition brought about by an increase in fluid shear that raises the Viscous number to the condition $I_{v}=I_{c g}$. Unfortunately, almost nothing is known about creep in mud. Finally, while the Boyer et al. (2011) rheology appears to capture some of the known behavior of mud, 
it does not explicitly incorporate cohesion. Interparticle attraction at high concentrations is common for natural clays and silts, and may have a strong influence on the observed rheology.

\subsection{Interactions and feedbacks between granular flows (II) and suspensions (I)}

Researchers are well aware that, in principle, bed and suspended load represent two limits of a continuum of fluid-driven sediment transport. In practice, however, we model them as two different processes. The physical basis for most modern bed-load transport equations is a momentum balance between the near-bed fluid and particles on the surface of the bed (Charru et al. 2004). For turbulent flows, this results in a relation between flux and excess fluid stress to a power of 3/2 (Lajeunesse et al., 2010, Meyer-Peter and Müller, 1948). The physical basis for suspended sediment transport is the Rouse profile, in which upward-directed turbulent diffusion of sediment is balanced by advective settling due to gravity. This formulation predicts a sediment concentration that decreases as a power-law function with elevation above the bed, and requires as a boundary condition that the elevation and concentration of the top of the bedload layer be specified. Both approaches have proven somewhat successful in their respective limits, however for mixed bed- and suspended-load transport we resort to a "total load equation" such as Engelund and Hansen (1967) where flux goes with fluid stress to a power of $5 / 2$. None of these frameworks explicitly incorporate momentum exchange from grain-grain collision, which we now know is an important component of bed load (Capart and Fraccarollo, 2011, Houssais et al. 2015).

The rheological framework presented and applied to laminar sediment transport above presents an appealing unification of bed and suspended load, because this is modeled as a continuous transition from a granular flow to a dilute suspension. Expansion of this framework into rivers, however, will require consideration of turbulence, collisions and heterogeneous particles. We expect bed-load rheology will be mostly unaffected by Reynolds number, since fluid stress (including turbulent stresses) decays rapidly as the particle concentration becomes significantly dense $(\Phi \geq 0.1)$ (Schmeeckle, 2014b, Wright and Parker, 2004), and experiments conducted by Capart and Fraccarollo (2011) with turbulent flows and non-spherical particles show similar behavior to our laminar experiments described above. Considering dilute suspension $(\Phi<0.1)$, however, the hydrodynamic stress profile is sufficiently different for the turbulent case relative to the laminar one that we expect the rheology would have to be modified. For laminar flows the dilute limit obeys the classical Einstein suspension rheology. The rheology of dilute turbulent flows is much less well known, but the effective viscosity must include an eddy viscosity and granular drag effects. It is known that suspended sediment itself can influence the eddy viscosity at only modest concentrations (Wright and Parker, 2004); once concentrations reach order 10\%, turbulence may be completely suppressed (Sequeiros et al., 2009). A unified rheological approach that accounts for these effects would be useful not only for fluvial transport, but also for modeling turbidity current flows. These currents are dilute suspensions, but also transport significant sediment at their base in the form of bed load (Sequeiros et al., 2010); thus, we expect a continuous transition from dense granular flow to suspension (II $\rightarrow$ I) from the base of the current upwards. The generation of turbidity currents by fluid entrainment from debris flows also represents a granular flow to suspension (II $\rightarrow$ I) transition.

\section{Opportunities and Challenges}

To summarize, our proposition is that a wide range of Earth-material flows, from landslides to mudflows to bed load to suspensions, form a continuum of transport regimes that may all be 


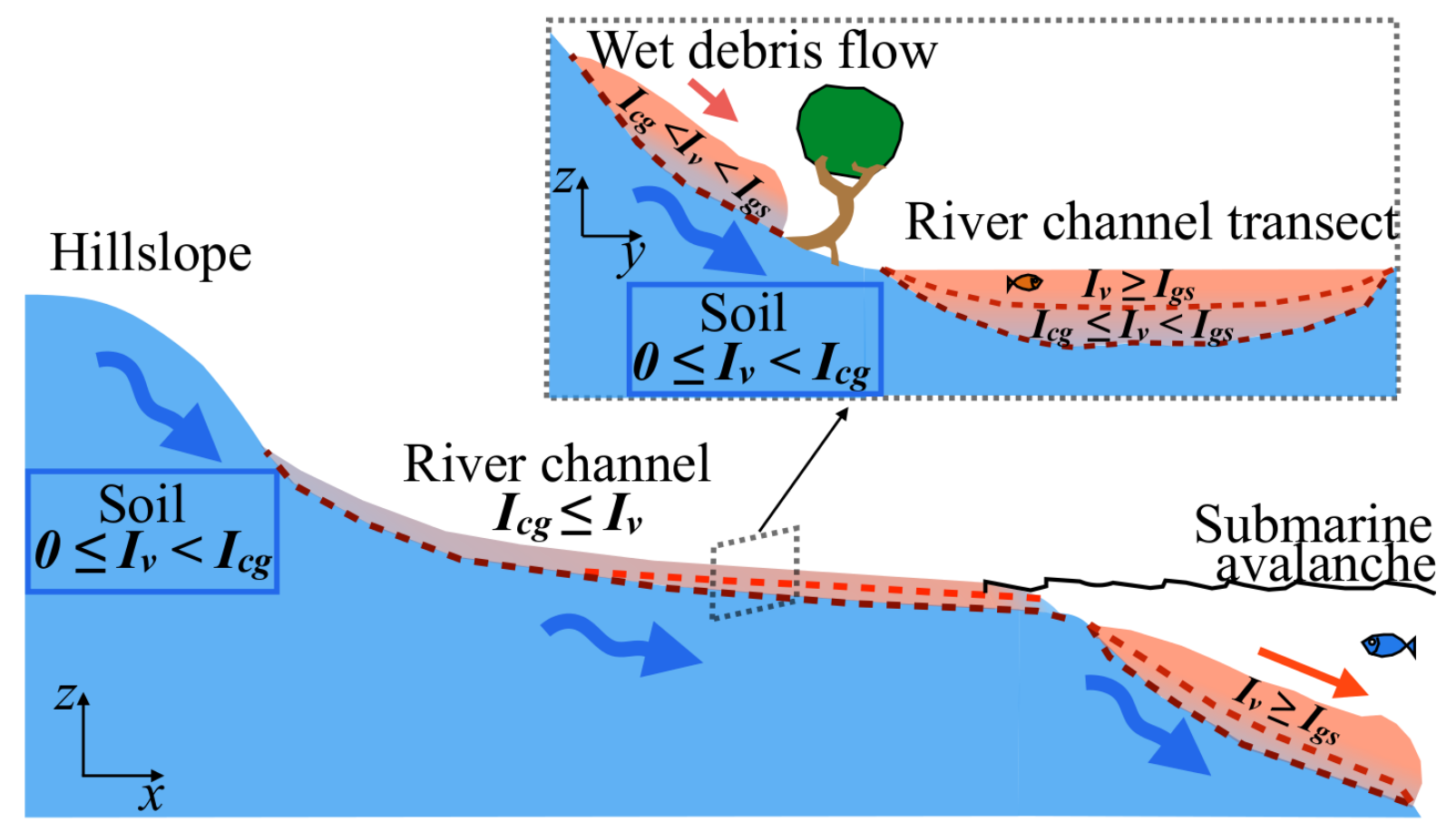

Figure 8: Sediment-composed landscape sketch where color represents the Viscous number. Red dashed lines emphasize regime transitions. $x, y$ and $z$ indicate directions of sediment flow, transverse to flow, and vertical, respectively. Main sketch represents a landscape profile from source (hillslope) to sink (ocean); inset shows a cross-section of a river and its valley.

described with a unified submerged granular rheology (Fig. 8). The current candidate model is the local $\mu\left(I_{v}\right)$ rheology proposed by Boyer et al. (2011) and verified by Houssais et al. (2016), which combines the known behaviors of dense granular flows and dilute suspensions. This model proposed, however, that $\mu \rightarrow \mu_{s}$ as $I_{v} \rightarrow 0$; i.e., that granular systems transition to a jammed state associated with static friction. This last point is incompatible with creep, and our new observations reveal that friction continues to decrease with decreasing Viscous number below the static value. Instead, we propose that the critical Viscous number $I_{c g}$ and the associated friction $\mu=\mu_{s}$ marks a transition between granular flow and creep. In this view the onset of bed-load transport, the fluidization of terrestrial and submarine landslides, and the inception of mudflows, are a continuous transition from quasi-static creep to a dense-granular flow. The lower bound on creep, however, is a complete unknown. Moreover, the rheology of the creep regime is not understood, although recent modeling suggests that the $\mu\left(I_{v}\right)$ framework may be extended to describe creep by inclusion of a nonlocal length scale for particle motion (Kamrin and Koval, 2012).

Returning to the idea of landscape evolution modeling, we believe that the continuous and reversible transitions among granular transport regimes present a challenge to the paradigm of dividing the environment up into different process domains. Instead, the movement of sediment can be related to an effective friction that increases from the hillslope to the river and downstream, as the Viscous number increases. The long profile of the hillslope-river system is a gradient of decreasing sediment:water above the surface $I_{v}=I_{c g}$ which, associated with the concomitant decay of particle size, results in an increase of the effective friction coefficient and the Viscous number of the sediment-fluid mixture. As a consequence, the proportion of the total sediment flux moving as suspension increases downstream. We envision an approach to modeling sediment transport at the landscape scale that embraces this continuity, and employs 
a unified rheology framework as its foundation (see figure 8). Such an approach is not without its challenges of course; new closure schemes will have to be introduced in order to have a full set of equations capable of producing a numerical value of flux for a given combination of gravity and fluid shear. If we take the Boyer et al. (2011) model at face value, knowledge of $I_{v}$ is sufficient to estimate $\mu$, and then we need only an estimate of the granular shear rate at the top of the flow to determine everything else. In practice, however, a priori prediction is challenging because the values for $I_{v}$ in natural settings are not prescribed by boundary conditions, but are themselves emergent from the transport system.

Although the "onset of motion" may be continuous and more complex than a simple critical stress, this concept is still useful for helping to understand the equilibrium shapes of landscapes such as dune-lee faces and hillslopes (Pouliquen, 1999), river-channel cross sections (Reitz et al. 2014, Seizilles et al. 2014) and river profiles (Devauchelle et al., 2011, Guerit et al., 2014, Miller et al. 2014, Parker, 1978). For all of these systems, in which transport is driven by a dense granular flow (including bed load), the hypothesis used is that the system shape evolves toward a limit where $\tau$ is equal or close to $\tau_{c}$ everywhere. This hypothesis is not incompatible with transport, so long as the transport layer remains thin $(\leq d)$. For fluvial bed-load transport, this condition means that the fluid shear stress still acts on a well-defined bed surface because the boundary between bed and fluid is sharp. Also, because the timescales of particle motion in bed load are short - typically the particle settling time (Lajeunesse et al., 2010) - compared to the timescale of bed evolution due to granular creep, the existence of creep alone likely has little influence on river morphodynamics. However, the "onset of motion" hypothesis for riverchannel geometry becomes problematic when the system evolves at high transport conditions, as recently confirmed from river field data (Gaurav et al. 2015). In this situation, a thick bed-load layer develops and the interface between pure fluid and sediment bed becomes fuzzy. Indeed, while the morphology of bed-load rivers is well understood through their organization to (near-)threshold conditions, we do not as yet have a theory for what sets the channel geometry of suspension rivers (Li et al., 2015). The sediment transport rheology (Fig. 5) may yet bring new insights to bear on this problem. In particular, the dynamics of the surface defined by $I_{v}=I_{c g}$ may be the most relevant to morphodynamics, even when additional transport occurs in suspension above that surface (see sketch fig. 8).

There are likely many more lessons to be learned by extending concepts from granular and soft-matter physics to geomorphology (Frey and Church, 2009, 2011). Linking sediment creep to glass dynamics, for example, could provide a formalism for assessing slow flow, aging and hysteresis, and phase transitions. Much as the framework of statistical mechanics has been recently adopted by Furbish and colleagues (Furbish et al., 2012) to link stochastic grain-scale motion to bulk (ensemble-averaged) flux, recent studies in the rheology of granular systems have developed a formalism for connecting particle-scale dynamics to the emergent constitutive laws that may be developed further in service of sediment transport. The general characteristics of regime transitions in disordered granular systems can provide a guideline for how to collect data. For example, the diverging effective viscosity of granular materials on approach to threshold means that the timescale needed to average over in order to determine a representative flux also grows rapidly (Houssais et al., 2015). This could help to explain the breakdown of bed-load transport equations in the vicinity of threshold (Recking et al. 2012), and could be used to develop a dynamically-informed measurement timescale for data collection.

Finally, our study highlights how geomorphology can also lead to the discovery of new phenomena that challenge and motivate further exploration in soft-matter physics. For example, the laminar sediment transport experiments exhibit all three granular regimes simultaneously, allowing us to probe the transitions across these regimes in unprecedented detail. The transition to creep challenges our understanding of jamming, and presents a new view on the triggering 
of granular avalanches. Finally, mudflows, pyroclastic flows, turbidity currents and bed load represent exotic states of particulate matter that are under studied in physics.

Table 1: Table of all variables and their units

\begin{tabular}{|c|c|c|}
\hline Symbol & Meaning & Units (SI) \\
\hline$\dot{\gamma} \equiv \dot{\gamma}_{x z}$ & Particle shear rate & $\mathrm{s}^{-1}$ \\
$\eta_{f}$ & Fluid viscosity & Pa.s \\
$\eta_{e f f}$ & Ea.s \\
$\theta$ & Slope angle & Rad \\
$\mu$ & Effective friction coefficient & - \\
$\mu_{s}$ & Static friction coefficient value & - \\
$\mu_{d}$ & Dynamic friction coefficient value & - \\
$\rho_{p}$ & Particle density & $\mathrm{kg} . \mathrm{m}^{-3}$ \\
$\rho$ & Fluid density & $\mathrm{kg} \cdot \mathrm{m}^{-3}$ \\
$\tau \tau_{x z}$ & Flow shear stress & $\mathrm{Pa}$ \\
$\tau_{c}$ & Critical (or yield) stress value & $\mathrm{Pa}$ \\
$\Phi$ & Packing fraction: volume fraction occupied by solid & - \\
$\Phi_{c}$ & Critical packing fraction value, associated with the transition to Jamming & - \\
$F_{/ /}$ & Total tangential force per unit of width & $\mathrm{N} . \mathrm{m}^{-1}$ \\
$F_{\perp}$ & Total normal force per unit of width & $\mathrm{N} . \mathrm{m}^{-1}$ \\
$F_{g}$ & Gravity force per unit of width & $\mathrm{N} . \mathrm{m}^{-1}$ \\
$F_{s}$ & Flow shear force per unit of width & $\mathrm{N} . \mathrm{m}^{-1}$ \\
$I$ & Inertial number $=\dot{\gamma} d \sqrt{\rho_{p}} / \sqrt{P_{p}}$ & - \\
$I_{v}$ & Viscous number $=\eta_{f} \dot{\gamma} / P_{p}$ & - \\
$I_{c g}$ & Critical viscous number value for the creep-to-granular flow transition & - \\
$I_{g s}$ & Critical viscous number value for the granular-to-suspension flow transition & - \\
$P_{p}$ & Confinement pressure & $\mathrm{Pa}$ \\
$P_{0}$ & Remaining normal stress at the bed-fluid interface, due to particle weight & $\mathrm{Pa}$ \\
$d$ & Particle diameter & $\mathrm{m}$ \\
$g$ & Gravity & $\mathrm{m} . \mathrm{s}^{-2}$ \\
$q_{s}$ & Sediment flux per unit of width \\
\hline
\end{tabular}


Acknowledgements: Research was supported by US Army Research Office - Division of Earth Materials and Processes grant 64455EV, and US National Science Foundation grant EAR-1224943 to D.J.J. We thank C. Ortiz and D.J Durian for their contributions to the original research that formed the starting point for this paper.

\section{References}

Ancey, C. (2007). Plasticity and geophysical flows: A review. J. Non-Newton. Fluid Mech., 142(1-3):4-35. WOS:000245609700002.

Anderson, R. S. and Anderson, S. P. (2010). Geomorphology: the mechanics and chemistry of landscapes. Cambridge University Press.

Aussillous, P., Chauchat, J., Pailha, M., Médale, M., and Guazzelli, E. (2013). Investigation of the mobile granular layer in bedload transport by laminar shearing flows. J. Fluid Mech., 736(2013):594-615.

Bagnold, R. (1966). An approach to the sediment transport problem. General Physics Geological Survey, Prof. paper.

Bagnold, R. a. (1954). Experiments on a Gravity-Free Dispersion of Large Solid Spheres in a Newtonian Fluid under Shear. Proc. R. Soc. A Math. Phys. Eng. Sci., 225(1160):49-63.

Bagnold, R. A. (1956). The flow of cohesionless grains in fluids. Philosophical Transactions of the Royal Society of London A: Mathematical, Physical and Engineering Sciences, 249(964):235297.

Balmforth, N. and Kerswell, R. (2005). Granular collapse in two dimensions. Journal of Fluid Mechanics, 538:399-428.

Barnes, H. A. (1999). The yield stress - a review or $\pi \alpha \nu \tau \alpha \rho \varepsilon \iota$ ' - everything flows? Journal of Non-Newtonian Fluid Mechanics, 81(1):133-178.

Barry, J. J., Buffington, J. M., and King, J. G. (2004). A general power equation for predicting bed load transport rates in gravel bed rivers. Water Resour. Res., 40(10):W10401.

Bouchez, J., Gaillardet, J., France-Lanord, C., Maurice, L., and Dutra-Maia, P. (2011). Grain size control of river suspended sediment geochemistry: Clues from amazon river depth profiles. Geochemistry, Geophysics, Geosystems, 12(3).

Boyer, F., Guazzelli, E., and Pouliquen, O. (2011). Unifying Suspension and Granular Rheology. Phys. Rev. Lett., 107(18):188301.

Buffington, J. M. and Montgomery, D. R. (1997). A systematic analysis of eight decades of incipient motion studies, with special reference to gravel-bedded rivers. Water Resour. Res., 33(8):1993-2029.

Capart, H. and Fraccarollo, L. (2011). Transport layer structure in intense bed-load. Geophysical Research Letters, 38(20).

Cassar, C., Nicolas, M., and Pouliquen, O. (2005). Submarine granular flows down inclined planes. Phys. Fluids, 17(10):103301. 
Charru, F., Mouilleron, H., and Eiff, O. (2004). Erosion and deposition of particles on a bed sheared by a viscous flow. J. Fluid Mech., 519(2004):55-80.

Chiodi, F., Claudin, P., and Andreotti, B. (2014). A two-phase flow model of sediment transport: transition from bedload to suspended load. J. Fluid Mech., 755:561-581.

Coussot, P. (1995). Structural Similarity and Transition from Newtonian to Non-Newtonian Behavior for Clay-Water Suspensions. Phys. Rev. Lett., 74(20):3971-3974.

Coussot, P. and Piau, J. (1994). On the Behavior of Fine Mud Suspensions. Rheol. Acta, 33(3):175-184. WOS:A1994NX68900003.

Culling, W. (1960). Analytical theory of erosion. The Journal of Geology, pages 336-344.

Dankers, P. J. T. and J. C. Winterwerp (2007). Hindered settling of mud flocs: Theory and validation. Continental Shelf Research, 27(14):1893-1907.

Devauchelle, O., Petroff, A., Lobkovsky, A., and Rothman, D. H. (2011). Longitudinal profile of channels cut by springs. Journal of Fluid Mechanics, 667:38-47.

Di Maio, C., Vassallo, R., and Vallario, M. (2013). Plastic and viscous shear displacements of a deep and very slow landslide in stiff clay formation. Engineering Geology, 162:53-66.

Dietrich, W. E., Bellugi, D. G., Sklar, L. S., Stock, J. D., Heimsath, A. M., and Roering, J. J. (2003). Geomorphic transport laws for predicting landscape form and dynamics. Prediction in geomorphology, pages 103-132.

Dijksman, J. a., Rietz, F., Lorincz, K. a., van Hecke, M., and Losert, W. (2012). Invited Article: Refractive index matched scanning of dense granular materials. Rev. Sci. Instrum., 83(1):011301.

Diplas, P., Dancey, C. L., Celik, A. O., Valyrakis, M., Greer, K., and Akar, T. (2008). The role of impulse on the initiation of particle movement under turbulent flow conditions. Science, 322(5902):717-720.

du Pont, S. C., Gondret, P., Perrin, B., and Rabaud, M. (2003). Granular avalanches in fluids. Physical Review Letters, 90(4):044301.

Durán, O., Andreotti, B., and Claudin, P. (2012). Numerical simulation of turbulent sediment transport, from bed load to saltation. Phys. Fluids, 24:103306.

Einstein, A. (1905). On the movement of small particles suspended in a stationary liquid demanded by the molecular-kinetic theory of heat. Annalen der physik, 17:549-560.

Eke, E., Viparelli, E., and Parker, G. (2011). Field-scale numerical modeling of breaching as a mechanism for generating continuous turbidity currents. Geosphere, 7(5):1063-1076.

Engelund, F. and Hansen, E. (1967). A monograph on sediment transport in alluvial streams. Technical report, TEKNISKFORLAG Skelbrekgade 4 Copenhagen V, Denmark.

Felix, M. and Peakall, J. (2006). Transformation of debris flows into turbidity currents: mechanisms inferred from laboratory experiments. Sedimentology, 53(1):107-123.

Forterre, Y. and Pouliquen, O. (2008). Flows of dense granular media. Annu. Rev. Fluid Mech., 40:1-24. 
Frey, P. and Church, M. (2009). How river beds move. Science, 325(5947):1509-10.

Frey, P. and Church, M. (2011). Bedload: a granular phenomenon. Earth Surf. Process. Landforms, 36(1):58-69.

Furbish, D. J., Haff, P. K., Roseberry, J. C., and Schmeeckle, M. W. (2012). A probabilistic description of the bed load sediment flux: 1. Theory. J. Geophys. Res., 117(F3):F03031.

Gaurav, K., Métivier, F., Devauchelle, O., Sinha, R., Chauvet, H., Houssais, M., and Bouquerel, H. (2015). Morphology of the kosi megafan channels. Earth Surface Dynamics, 3(3):321-331.

Gravish, N. and Goldman, D. I. (2014). Effect of volume fraction on granular avalanche dynamics. Physical Review E, 90(3):032202.

Guerit, L., Métivier, F., Devauchelle, O., Lajeunesse, E., and Barrier, L. (2014). Laboratory alluvial fans in one dimension. Physical Review E, 90(2):022203.

Ham, J. M. and Homsy, G. M. (1988). Hindered settling and hydrodynamic dispersion in quiescent sedimenting suspensions. International Journal of Multiphase Flow, 14(5):533-546.

Houssais, M. and Lajeunesse, E. (2012). Bedload transport of a bimodal sediment bed. $J$. Geophys. Res., 117:F04015.

Houssais, M., Ortiz, C. P., Durian, D. J., and Jerolmack, D. J. (2015). Onset of sediment transport is a continuous transition driven by fluid shear and granular creep. Nature Communications, 6 .

Houssais, M., Ortiz, C. P., Durian, D. J., and Jerolmack, D. J. (2016). Rheology of sediment transported by a laminar flow. under review at Physics of Fluids.

Hsu, L., Finnegan, N. J., and Brodsky, E. E. (2011). A seismic signature of river bedload transport during storm events. Geophysical Research Letters, 38(13).

Imran, J., Harff, P., and Parker, G. (2001). A numerical model of submarine debris flow with graphical user interface. Computers $\&$ geosciences, 27(6):717-729.

Iverson, R., Reid, M., Iverson, N., LaHusen, R., Logan, M., Mann, J., and Brien, D. (2000). Acute sensitivity of landslide rates to initial soil porosity. Science, 290(5491):513-516.

Iverson, R. M. (1997). The physics of debris flows. Rev. Geophys., 35(3):245-296. WOS:A1997XN90100002.

Iverson, R. M. and George, D. L. (2014). A depth-averaged debris-flow model that includes the effects of evolving dilatancy. i. physical basis. Proc. R. Soc. A, 470(3):032202.

Jaeger, H. M., Nagel, S. R., and Behringer, R. P. (1996). Granular solids, liquids, and gases. Rev. Mod. Phys., 68(4):1259-1273.

Jerolmack, D. J. (2011). Causes and effects of noise in landscape dynamics. EOS, Trans. AGU, 92(44):385-386.

Jop, P., Forterre, Y., and Pouliquen, O. (2005). Crucial role of sidewalls in granular surface flows: consequences for the rheology. Journal of Fluid Mechanics, 541:167-192.

Jop, P., Forterre, Y., and Pouliquen, O. (2006). A constitutive law for dense granular flows. Nature, 441(7094):727-30. 
Kamrin, K. and Koval, G. (2012). Nonlocal constitutive relation for steady granular flow. Physical Review Letters, 108(17):178301.

Keys, A. S., Abate, A. R., Glotzer, S. C., and Durian, D. J. (2007). Measurement of growing dynamical length scales and prediction of the jamming transition in a granular material. Nature physics, 3(4):260-264.

Knight, J. B., Fandrich, C. G., Lau, C. N., Jaeger, H. M., and Nagel, S. R. (1995). Density relaxation in a vibrated granular material. Physical review E, 51(5):3957.

Komatsu, T., Inagaki, S., Nakagawa, N., and Nasuno, S. (2001). Creep Motion in a Granular Pile Exhibiting Steady Surface Flow. Phys. Rev. Lett., 86(9):1757-1760.

Koval, G., Roux, J.-N., Corfdir, A., and Chevoir, F. (2009). Annular shear of cohesionless granular materials: From the inertial to quasistatic regime. Physical Review E, 79(2):021306.

Kranenburg, C. (1999). Effects of floc strength on viscosity and deposition of cohesive sediment suspensions. Continental Shelf Research, 19(13):1665-1680.

Lagrée, P.-Y., Staron, L., and Popinet, S. (2011). The granular column collapse as a continuum: validity of a two-dimensional navier-stokes model with a $\mu$ (i)-rheology. Journal of Fluid Mechanics, 686:378-408.

Lajeunesse, E., Malverti, L., and Charru, F. (2010). Bed load transport in turbulent flow at the grain scale: Experiments and modeling. J. Geophys. Res., 115(F4):F04001.

Lajeunesse, E., Mangeney-Castelnau, A., and Vilotte, J. (2004). Spreading of a granular mass on a horizontal plane. Physics of Fluids (1994-present), 16(7):2371-2381.

Li, C., Czapiga, M. J., Eke, E. C., Viparelli, E., and Parker, G. (2015). Variable shields number model for river bankfull geometry: bankfull shear velocity is viscosity-dependent but grain size-independent. Journal of Hydraulic Research, 53(1):36-48.

Liu, A. J. and Nagel, S. R. (2010). The jamming transition and the marginally jammed solid. Annu. Rev. Condens. Matter Phys., 1(1):347-369.

Lube, G., Huppert, H. E., Sparks, R. S. J., and Hallworth, M. A. (2004). Axisymmetric collapses of granular columns. Journal of Fluid Mechanics, 508:175-199.

Mackey, B. H. and Roering, J. J. (2011). Sediment yield, spatial characteristics, and the longterm evolution of active earthflows determined from airborne lidar and historical aerial photographs, eel river, california. Geological Society of America Bulletin, 123(7-8):1560-1576.

Major, J. and Pierson, T. (1992). Debris Flow Rheology - Experimental-Analysis of FineGrained Slurries. Water Resour. Res., 28(3):841-857. WOS:A1992HG17500024.

Malverti, L., Lajeunesse, E., and Métivier, F. (2008). Small is beautiful: Upscaling from microscale laminar to natural turbulent rivers. Journal of Geophysical Research: Earth Surface (2003-2012), 113(F4).

Mari, R., Krzakala, F., and Kurchan, J. (2009). Jamming versus glass transitions. Physical review letters, 103(2):025701.

Mari, R., Seto, R., Morris, J. F., and Denn, M. M. (2014). Shear thickening, frictionless and frictional rheologies in non-brownian suspensions. Journal of Rheology (1978-present), 58(6):1693-1724. 
Mari, R., Seto, R., Morris, J. F., and Denn, M. M. (2015). Discontinuous shear thickening in brownian suspensions by dynamic simulation. Proceedings of the National Academy of Sciences, 112(50):15326-15330.

Mastbergen, D. R. and Van Den Berg, J. H. (2003). Breaching in fine sands and the generation of sustained turbidity currents in submarine canyons. Sedimentology, 50(4):625-637.

Mehta, A.J., Hayter, E.J., Parker, W.R., Krone, R.B., and Teeter, A.M. (1989). Cohesive Sediment Transport. I: Process Description. Journal of Hydraulic Engineering, 115(8):10761093.

Meyer-Peter, E. and Müller, R. (1948). Formulas for bed-load transport. In Stockholm, S., editor, Proceedings, 2nd Congress, International Association of Hydraulic Research, pages $39-64$.

MiDi, G. (2004). On dense granular flows. Eur. Phys. J. E, 14:341-365.

Miller, K. L., Reitz, M. D., and Jerolmack, D. J. (2014). Generalized sorting profile of alluvial fans. Geophysical Research Letters, 41(20):7191-7199.

Mitchell, J. K. a. (1993). Fundamentals of soil behavior. Wiley New York.

Nguyen, V. B., Darnige, T., Bruand, A., Clement, E., et al. (2011). Creep and fluidity of a real granular packing near jamming. Physical review letters, 107(13):138303.

Nichol, K., Zanin, A., Bastien, R., Wandersman, E., and van Hecke, M. (2010). Flow-Induced Agitations Create a Granular Fluid. Phys. Rev. Lett., 104(7):078302.

O'Brien, J. S. and Julien, P. Y. (1988). Laboratory Analysis of Mudflow Properties. Journal of Hydraulic Engineering, 114(8):877-887.

Ouriemi, M., Aussillous, P., and Guazzelli, E. (2009). Sediment dynamics. Part 1. Bed-load transport by laminar shearing flows. J. Fluid Mech., 636(2009):295.

Pailha, M., Nicolas, M., and Pouliquen, O. (2008). Initiation of underwater granular avalanches: Influence of the initial volume fraction. Physics of Fluids (1994-present), 20(11):111701.

Pailha, M. and Pouliquen, O. (2009). A two-phase flow description of the initiation of underwater granular avalanches. Journal of Fluid Mechanics, 633:115-135.

Parchure, T.M. and Mehta, A.J. (1985). Erosion of Soft Cohesive Sediment Deposits. Journal of Hydraulic Engineering, 111(10):1308-1326.

Parker, G. (1978). Self-formed straight rivers with equilibrium banks and mobile bed. part 2 . the gravel river. Journal of Fluid Mechanics, 89(01):127-146.

Parker, G., Fukushima, Y., and Pantin, H. M. (1986). Self-accelerating turbidity currents. Journal of Fluid Mechanics, 171:145-181.

Perron, J. T., Kirchner, J. W., and Dietrich, W. E. (2009). Formation of evenly spaced ridges and valleys. Nature, 460:502-505.

Pons, A., Amon, A., Darnige, T., Crassous, J., and Clément, E. (2015). Mechanical fluctuations suppress the threshold of soft-glassy solids: the secular drift scenario. Physical Review E. 
Pouliquen, O. (1999). Scaling laws in granular flows down rough inclined planes. Physics of Fluids (1994-present), 11(3):542-548.

Recking, A., Liébault, F., Peteuil, C., and Jolimet, T. (2012). Testing bedload transport equations with consideration of time scales. Earth Surf. Process. Landforms, 37(7):774-789.

Reddy, K. A., Forterre, Y., and Pouliquen, O. (2011). vidence of mechanically activated processes in slow granular flows. Physical Review Letters, 106(10):108301.

Reitz, M. D., Jerolmack, D. J., Lajeunesse, E., Limare, A., Devauchelle, O., and Métivier, F. (2014). Diffusive evolution of experimental braided rivers. Physical Review E, 89(5):052809.

Revil-Baudard, T. and Chauchat, J. (2013). A two-phase model for sheet flow regime based on dense granular flow rheology. Journal of Geophysical Research: Oceans, 118(2):619-634.

Revil-Baudard, T., Chauchat, J., Hurther, D., and Barraud, P.-A. (2015). Investigation of sheet-flow processes based on novel acoustic high-resolution velocity and concentration measurements. Journal of Fluid Mechanics, 767:1-30.

Rijn, L. C. v. (1984). Sediment transport, part ii: suspended load transport. Journal of hydraulic engineering, 110(11):1613-1641.

Roche, O. (2012). Depositional processes and gas pore pressure in pyroclastic flows: an experimental perspective. Bulletin of volcanology, 74(8):1807-1820.

Roche, O., Gilbertson, M., Phillips, J., and Sparks, R. (2004). Experimental study of gasfluidized granular flows with implications for pyroclastic flow emplacement. Journal of Geophysical Research: Solid Earth (1978-2012), 109(B10).

Roche, O., Montserrat, S., Niño, Y., and Tamburrino, A. (2008). Experimental observations of water-like behavior of initially fluidized, dam break granular flows and their relevance for the propagation of ash-rich pyroclastic flows. Journal of Geophysical Research: Solid Earth (1978-2012), 113(B12).

Roering, J. J., Kirchner, J. W., and Dietrich, W. E. (1999). Evidence for nonlinear, diffusive sediment transport on hillslopes and implications for landscape morphology. Water Resour. Res., 35(3):853-870.

Roering, J. J., Kirchner, J. W., Sklar, L. S., and Dietrich, W. E. (2001). Hillslope evolution by nonlinear creep and landsliding: An experimental study. Geology, 29(2):143-146.

Rondon, L., Pouliquen, O., and Aussillous, P. (2011). Granular collapse in a fluid: role of the initial volume fraction. Physics of Fluids (1994-present), 23(7):073301.

Roseberry, J. C., Schmeeckle, M. W., and Furbish, D. J. (2012). A probabilistic description of the bed load sediment flux: 2. Particle activity and motions. J. Geophys. Res., 117(F3):F03032.

Savage, S., Babaei, M., and Dabros, T. (2014). Modeling gravitational collapse of rectangular granular piles in air and water. Mechanics Research Communications, 56:1-10.

Schmeeckle, M. W. (2014a). Numerical simulation of turbulence and sediment transport of medium sand. Journal of Geophysical Research: Earth Surface, 119(6):1240-1262.

Schmeeckle, M. W. (2014b). Numerical simulation of turbulence and sediment transport of medium sand. J. Geophys. Res. Earth Surf., 119(6):1240-1262. 
Seizilles, G., Lajeunesse, E., Devauchelle, O., and Bak, M. (2014). Cross-stream diffusion in bedload transport. Physics of Fluids (1994-present), 26(1):013302.

Sequeiros, O. E., Naruse, H., Endo, N., Garcia, M. H., and Parker, G. (2009). Experimental study on self-accelerating turbidity currents. Journal of Geophysical Research: Oceans (19782012), 114(C5).

Sequeiros, O. E., Spinewine, B., Beaubouef, R. T., Sun, T., García, M. H., and Parker, G. (2010). Characteristics of velocity and excess density profiles of saline underflows and turbidity currents flowing over a mobile bed. Journal of Hydraulic Engineering, 136(7):412-433.

Shields, I. A. (1936). Anwendung der ahnlichkeitmechanik und der turbulenzforschung auf die gescheibebewegung. Mitt. Preuss Ver.-Anst., 26.

Singh, A., Magnanimo, V., Saitoh, K., and Luding, S. (2015). The role of gravity or pressure and contact stiffness in granular rheology. New Journal of Physics, 17(4):043028.

Smith, T. R. and Bretherton, F. P. (1972). Stability and the conservation of mass in drainage basin evolution. Water Resources Research, 8(6):1506-1529.

Stickel, J. J. and Powell, R. L. (2005). Fluid mechanics and rheology of dense suspensions. Annu. Rev. Fluid Mech., 37:129-149.

Talling, P. J., Masson, D. G., Sumner, E. J., and Malgesini, G. (2012). Subaqueous sediment density flows: depositional processes and deposit types. Sedimentology, 59(7):1937-2003.

Traykovski, P., Geyer, W. R., Irish, J. D., and Lynch, J. F. (2000). The role of wave-induced density-driven fluid mud flows for cross-shelf transport on the Eel River continental shelf. Cont. Shelf Res., 20(16):2113-2140. WOS:000165362400004.

Turowski, J. M., Badoux, A., and Rickenmann, D. (2011). Start and end of bedload transport in gravel-bed streams. Geophys. Res. Lett., 38(4):L04401.

Vyalov, S. S. (1986). Rheological fundamentals of soil mechanics. Elsevier.

Wiberg, P. L. and Smith, J. D. (1987). Calculation of the Critical Shear Stress for Motion of Uniform and Heterogeneous Sediments. Water Resour. Res., 23(8):1471-1480.

Winterwerp, J. C. (2002). On the flocculation and settling velocity of estuarine mud. Cont. Shelf Res., 22(9):1339-1360. WOS:000176272000005.

Wong, M. and Parker, G. (2006). Reanalysis and correction of bed-load relation of meyer-peter and müller using their own database. Journal of Hydraulic Engineering, 132(11):1159-1168.

Wright, S. and Parker, G. (2004). Flow resistance and suspended load in sand-bed rivers: simplified stratification model. Journal of Hydraulic Engineering, 130(8):796-805.

Wyart, M. and Cates, M. (2014). Discontinuous shear thickening without inertia in dense non-brownian suspensions. Physical review letters, 112(9):098302.

$\mathrm{Xu}$, J. (2010). Normalized velocity profiles of field-measured turbidity currents. Geology, $38(6): 563-566$.

You, Y., Flemings, P., and Mohrig, D. (2012). Dynamics of dilative slope failure. Geology, 40(7):663-666. 
1006

1007

1008

1009

1010

You, Y., Flemings, P., and Mohrig, D. (2014a). Mechanics of dual-mode dilative failure in subaqueous sediment deposits. Earth and Planetary Science Letters, 397:10-18.

You, Y., Flemings, P., Mohrig, D., and Germaine, J. (2014b). How heterogeneity in the shear dilation of a deposit controls the mechanics of breaching slope failure. Journal of Geophysical Research: Earth Surface, 119(11):2381-2395. 